\title{
Generalizing Demonstrated Motion Trajectories using Coordinate-free Shape Descriptors
}

\author{
Maxim Vochten ${ }^{a}$, Tinne De Laet ${ }^{b}$ and Joris De Schutter ${ }^{a}$ \\ ${ }^{a}$ Department of Mechanical Engineering, KU Leuven \& Flanders Make, 3001 Leuven, Belgium \\ ${ }^{b}$ Faculty of Engineering Science, KU Leuven, 3001 Leuven, Belgium
}

\section{ARTICLE INFO}

\section{Keywords:}

Learning from demonstration

Trajectory generalization

Invariant shape descriptors

Constraint-based optimization

\begin{abstract}
A B S TR ACT
In learning by demonstration, the generalization of motion trajectories far away from the set of demonstrations is often limited by the dependency of the learned models on arbitrary coordinate references. Trajectory shape descriptors have the potential to remove these dependencies by representing demonstrated trajectories in a coordinate-free way. This paper proposes a constraint-based optimization framework to generalize demonstrated rigid-body motion trajectories to new situations starting from the shape descriptor of the demonstration. Experimental results indicate excellent generalization capabilities showing how, starting from only a single demonstration, new trajectories are easily generalized to novel situations anywhere in task space, such as new initial or target positions and orientations, while preserving similarity with the demonstration. The results encourage the use of trajectory shape descriptors in learning by demonstration to reduce the number of required demonstrations.
\end{abstract}

\section{Introduction}

In robot learning by demonstration, generalization refers to the transfer of what was learned from human demonstrations to executions on the robot in novel situations $[9,5]$. Generalization can be considered at different levels. At a higher level, task generalization involves recognizing the sequence of sub-tasks in a demonstrated task. The sub-tasks are often considered to be already known and available to the robot in a library of motion primitives [36, 18, 34]. Adapting the task to a new situation corresponds to stringing together the correct sequence of sub-tasks.

This paper instead deals with generalization at a lower level, trajectory generalization, meaning the adaptation of demonstrated motion trajectories to different task requirements, such as a different target location, a different motion profile or the avoidance of obstacles in the environment. Concretely, the focus is on rigid-body trajectories, which include many motions of practical interest, such as those of manipulated objects (pouring a jug, scooping with a spatula, stirring with a spoon...), the motion of the end effector of a robot manipulator, and the motion of human body segments. Therefore, we consider not only the translation of the object, but also its rotation.

One of the main challenges in learning by demonstration is the generalization of demonstrations to situations that are far away from the set of demonstrations in terms of space and time, while still preserving similarity with the demonstration. New situations may occur because of changes in task requirements (e.g. generalizing a pouring motion to a new glass at a different location), a different robot platform, or changes in the environment (e.g. obstacles to avoid or humans to interact with). An important reason for the often difficult generalization is that motions models are typically

@ maxim.vochten@kuleuven.be (M. Vochten); tinne.delaet@kuleuven. be (T. De Laet); joris. deschutter@kuleuven. be (J. De Schutter) constructed from trajectory coordinates that depend on arbitrary reference choices for the coordinates during recording. Examples include the choice of reference frame in which the motion coordinates are expressed, the choice of object frame that is used to track the manipulated object, the location and direction in space where the motion is taking place, or the chosen timing (velocity profile and duration) along the trajectory. Variation in these choices all have an influence on the measured trajectory coordinates, making the motion model dependent on these choices and hence limiting its ability to generalize. To deal with these cases, learning methods often need extra data in the form of additional demonstrations which limits the speed with which new skills may be learned. These dependencies can be partially compensated by applying a global transformation on the model to fit it to the new situation. However, such a transformation is not always uniquely defined or can be impossible to find depending on the given task requirements.

\subsection{Objective and approach}

The objective of this paper is to present a framework for generalizing demonstrated motion trajectories to new situations, while not being dependent on the need to encode arbitrary coordinate dependencies in the motion models. To do this, we first transform the demonstrated trajectory to a coordinate-free trajectory shape descriptor, prior to building the motion model. These shape descriptors provide a representation of the trajectory that can be independent of the choice for world reference frame, object references, velocity profile, duration, and geometric scale. Motion models constructed from the shape descriptors thus become more generally valid. Shape descriptors have already proven their use in motion recognition [41, 26, 45], reducing the search space during classification by eliminating unwanted coordinate variations, and hence resulting in an improved recognition rate. In this paper, the goal is to apply the shape descriptor approach to the problem of motion generalization. 


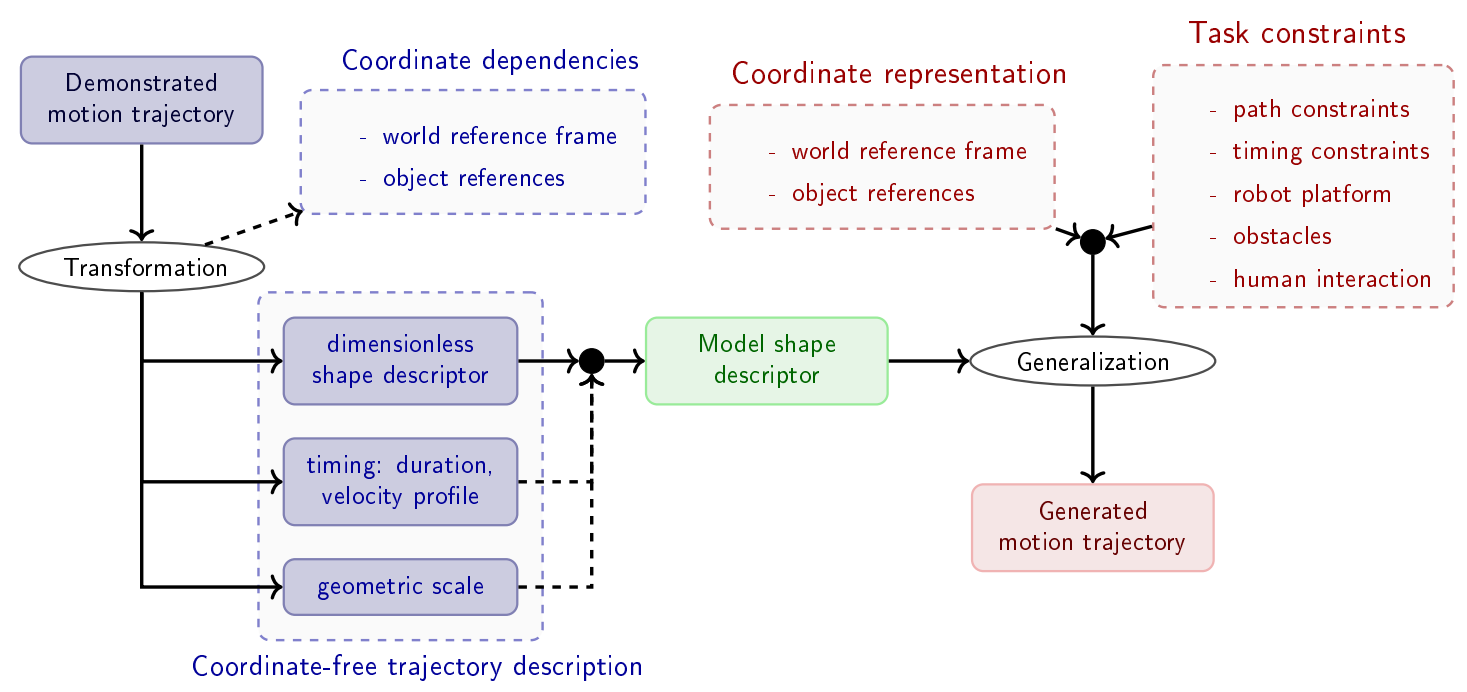

Figure 1: Overview of the proposed trajectory generalization approach. Demonstrated motion trajectories are first transformed to a coordinate-free description, which is further split up into a dimensionless shape descriptor, the timing information and the geometric scale. A model is built from the shape descriptor, possibly including the scale and timing of the demonstration. New trajectories are generated from the model shape descriptor by constraint-based optimization, where additional task requirements are imposed as constraints which are allowed to be expressed in different coordinate frames compared to the demonstration.

The shape descriptor approach is also motivated by research in human motion perception and generation [7]. Humans seem to have no problem to recognize and execute motions under different kinds of transformations. For example, when a motion is performed in a different direction, it is still perceived as the same motion, though the Cartesian trajectory coordinates would differ. Maintaining these invariant features when generating robot trajectories is therefore especially useful in human-robot interaction and collaboration applications, since the generated motion trajectories remain more natural and interpretable by the human. In addition, fewer demonstrations are needed to construct the motion model, since the shape descriptor is valid under different transformations of the original motion trajectory.

Figure 1 visualizes the developed generalization approach. The demonstrated trajectory is first transformed to a description that is coordinate-free. During this process, motion information is extracted related to reference choices, timing, and geometric scale. What remains is a dimensionless shape descriptor. A generative motion model is built from the shape descriptor, possibly including timing information and scale, if they are considered relevant to the application. During generalization, task requirements of the new situation, which may be expressed in different coordinate frames, are combined with the shape descriptor of the demonstration. These new task requirements may include a new initial or target location of the trajectory, timing constraints, workspace limits of the robot platform or obstacle avoidance. New trajectories are generated using constraint-based optimization, where the new task requirements are imposed as constraints and shape-similarity is preserved by minimizing deviation from the shape descriptor of the demonstration.

\subsection{Paper contributions and outline}

This paper has three main contributions. The first contribution is the mathematical formulation of the generative equations that define the rigid-body trajectory as a function of a generic coordinate-free shape descriptor. This compact, generic set of equations is elaborated for two types of existing shape descriptors: the extended Frenet-Serret invariants $[41,26]$ and the screw axis invariants [16].

The second contribution is the methodology for generalizing motion trajectories to new situations using constraintbased programming starting from the shape descriptor of the demonstration. New task requirements are imposed as constraints in the optimization problem, while deviation from the shape descriptor of the demonstration is minimized to preserve shape-similarity in the new execution.

The third contribution is an experimental verification of the proposed generalization method. It is shown how a demonstrated trajectory can be easily generalized to many new situations that are far from the demonstration in task space. A comparison is also made with two state-of-theart approaches for trajectory generalization not using shape descriptors: the dynamic movement primitives [21] and jerkaccuracy optimization [28].

Preliminary work on this topic was introduced in [42]. The first major extension in this paper is the abstraction of the generalization method from a specific shape descriptor (extended Frenet-Serret invariants) to a generic shape descriptor, making the methodology applicable to any other shape descriptor with a moving frame approach. The second extension is that more constraints are considered in the constraint-based optimization method for generalization, such as robot workspace limits and obstacle avoidance. Furthermore, the implementation of the optimization problem was 
made more efficient, e.g. by using a geometric integrator for the dynamics equations so that the properties of the rotation group are preserved. Additional experiments illustrate the ability of shape descriptors to generalize far away from the demonstrations, and includes an additional comparison with jerk-accuracy optimization [28].

The outline of the remainder of the paper is as follows. Section 2 provides on overview of related work on trajectory generalization in learning. Section 3 reviews different coordinate-free shape descriptors for rigid-body motion trajectories and establishes a generic formulation of the generative equations that reconstruct the motion trajectory from the shape descriptor. This generic formulation is used to formulate the constraint-based optimization method for trajectory generalization in Section 4, where also further details are given on the implementation and solution. Experiments in Section 5 illustrate how a demonstrated trajectory can be adapted to many different situations using the proposed generalization method, while similarity with what was demonstrated is preserved. This section also compares the method with other trajectory generalization approaches not using shape descriptors. Finally, Section 6 provides a discussion on the results and Section 7 concludes the paper.

\section{Related work on trajectory generalization}

The following approaches are commonly used for trajectory generalization in learning.

Spline-based approaches represent demonstrated trajectories using splines $[3,39]$. Trajectories are adapted to new situations by changing the weights on the basis functions or by superposing the trajectory with a polynomial function that has the desired continuity conditions at the start and end. While simple to implement, these methods may suffer from large undulations around the spline knots.

Probabilistic approaches generalize demonstrated motions by modeling the variability in repeatedly executed demonstrations of the motion. Trajectories are typically represented as the weighted sum of a set of basis functions. To adapt the trajectory to new situations, the weights on the basis functions are changed to fit to new situations. Hidden Markov Models (HMM) are used in [40, 6] to model the set of demonstrated trajectories, in which identified key points indicate the transition between the states in the HMM. Trajectories are reproduced by interpolating between these key points. In [13], the set of demonstrated trajectories is modeled using Gaussian Mixture Modeling (GMM), which can be extended to include orientation as well [23]. A smoothed generalized version of the trajectory is retrieved using Gaussian mixture regression. The Probabilistic Movement Primitives (ProMP) [30] represent trajectories as probability distributions allowing probabilistic operations on the underlying movement primitives such as blending or continuously switching from one motion primitive to the other. A disadvantage of probabilistic approaches is that multiple demonstrations are needed to calculate the probability distributions and that extrapolating outside of the learned distribution of demonstrations typically yields poor results.

Dynamical-system approaches focus on providing robustness in the presence of perturbations such as changes in the target position or obstacles along the path. The $D y$ namic Movement Primitives (DMP) [21] represent trajectories as dynamical systems consisting of the sum of a stable global attractor and a forcing term encoding the trajectory as a weighted sum of pre-defined basis functions. The global attractor provides the means to generalize to new target locations. Extensions have been made for including orientation $[31,24]$. These dynamical systems may however experience instability problems or convergence to other locations in state space when trying to reach a given target location. Other dynamical system approaches like $[22,35]$ focus on learning autonomous dynamical systems with guaranteed asymptotic stability properties. Even so, the generated trajectories lose more and more similarity with the demonstrated trajectories when moving away from the demonstrations in state space.

Global transformation approaches apply a transformation on (part of) the trajectory in order to change it to new situations. In [20], a rotation and translation is applied to generated trajectories to adapt to different start and end points, preserving the shape of the trajectory. In [33] trajectories are deformed using an affine transformation to change the trajectory to novel target points and to avoid obstacles, while preserving affine-invariant features. If the constraints of the new situation are too stringent however, it is possible that such a global transformation cannot be found. Related to this is task-parameterized GMM [11] where motion models are simultaneously learned in multiple reference frames from the demonstrated data. During generalization, the models are globally transformed to the newly supplied reference frames. Also here, the choice of reference frames is important and the transformations need to be explicitly stated.

Optimal control-based approaches solve an optimal control problem to adapt trajectories to new situations. In the objective function a cost term is introduced to preserve similarity with the original demonstration. For example in [32], this cost is the deviation of the motion profile (velocity, acceleration or jerk) of the generated trajectory to the motion profile of the original trajectory. In human motor control, the jerk is often minimized along the trajectory in order to obtain the typical bell-shaped motion profile with which humans execute their motions. The jerk-accuracy model in [28] extends this minimum-jerk cost with an accuracy cost that expresses the error with respect to the original trajectory in task space. Experimental results [27] indicate that the positional accuracy and velocity profile with respect to the demonstration are well preserved under trajectory perturbations compared to other methods such as DMP and ProMP.

Common to all these approaches is that generalizing farther away from the set of demonstrations typically yields worse results in terms of similarity to the demonstration. One of the main limiting factors is that the measured trajectory coordinates of the demonstrations depend on the arbitrary choice of references during recording, such as the cho- 
sen coordinate frames and the location and direction of the motion in space. Changes in these choices affect the coordinates while the motion remains inherently the same. This dependency on reference choices can be partially compensated by applying a global transformation on the trajectory coordinates to fit it to new task requirements, e.g. a new target position. However, such a transformation is not always uniquely defined or can be impossible to find depending on the new task requirements. Therefore, to improve generalization capabilities, this paper calculates a coordinate-free shape descriptor of the demonstration that is independent of these reference choices.

\section{Coordinate-free shape descriptors}

This section reviews two coordinate-free shape descriptors for rigid-body trajectories: the Screw Axis Invariants (SAI) and the extended Frenet-Serret Invariants (eFSI). For both descriptors, the generative equations are provided that allow reconstructing the object trajectory from the shape descriptor. We derive a general formulation of these generative equations by observing similarities in the equations of the two shape descriptors. This general formulation will be used in Section 4 to generalize trajectories to new situations using constraint-based optimization. The main results of this section are summarized in Table 1 on page 7.

\subsection{Fundamentals of rigid-body motion}

Before discussing the shape descriptors, we briefly review the position and velocity kinematics of rigid bodies.

\subsubsection{Position kinematics}

The spatial position of a rigid body can be characterized by rigidly attaching an orthogonal frame $\{o b j\}$ at a certain point of the body, and then registering the frame's orientation and position with respect to the world reference frame $\{w\}$. The orientation of $\{o b j\}$ with respect to $\{w\}$ is defined here using the $3 \times 3$ rotation matrix ${ }_{w}^{o b j} \boldsymbol{R}$. The position of $\{o b j\}$ is noted with the $3 \times 1$ vector ${ }_{w}^{o b j}$, which is the displacement vector from the origin of $\{w\}$ to the origin of $\{a b j\}$, as shown in Figure 2. Position and orientation together constitute the pose of the rigid body. The pose can be defined mathematically by the $4 \times 4$ pose matrix

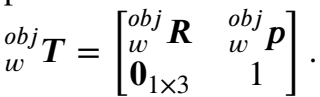

The rigid-body trajectory between the start of the motion $t_{0}$ and the end of the motion $t_{f}$ is then the time sequence of poses ${ }_{w}^{o b j} \boldsymbol{T}(t)$ for $t \in\left[t_{0}, t_{f}\right]$. Note that the motion trajectory coordinates in ${ }_{w}^{o b j} \boldsymbol{T}(t)$ may vary depending on the arbitrary choice of reference frames $\{o b j\}$ and $\{w\}$, even if the motion itself remains the same. The coordinate-free shape descriptors explained below aim to resolve this.

\subsubsection{Velocity kinematics}

The velocity of a rigid body is completely defined by the rotational velocity vector $\omega$ and the translational velocity vector $\boldsymbol{v}$ of a chosen point on the rigid body. If this chosen

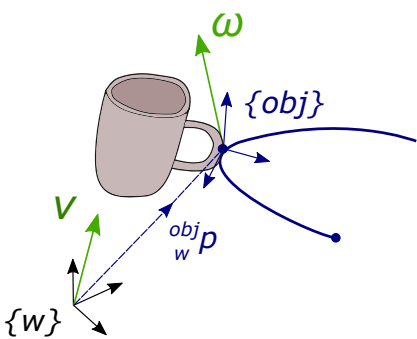

Figure 2: Illustration of how the position of a rigid body with respect to the world $\{w\}$ can be represented using an object frame $\{o b j\}$ with displacement vector ${ }_{w}^{o b j}$. The velocity of the rigid body can be defined by the rotational velocity vector $\omega$ and the translational velocity vector $\mathbf{v}$ in the origin of $\{w\}$.

point instantaneously coincides with the origin of the world reference frame $\{w\}$ (as visualized in Figure 2), and if the coordinates of $\boldsymbol{\omega}=\left[\omega_{x} \omega_{y} \omega_{z}\right]^{T}$ and $\boldsymbol{v}=\left[\begin{array}{lll}v_{x} & v_{y} & v_{z}\end{array}\right]^{T}$ are expressed in the body frame $\{o b j\}$, then the time-derivative of ${ }_{w}^{o b j} \boldsymbol{T}$, denoted as ${ }_{w}^{o b j} \boldsymbol{T}^{\prime}$, is defined as follows [29]:

$$
{ }_{w}^{o b j} \boldsymbol{T}^{\prime}={ }_{w}^{o b j} \boldsymbol{T}\left[\begin{array}{cccc}
0 & -\omega_{z} & \omega_{y} & v_{x} \\
\omega_{z} & 0 & -\omega_{x} & v_{y} \\
-\omega_{y} & \omega_{x} & 0 & v_{z} \\
0 & 0 & 0 & 0
\end{array}\right] .
$$

In terms of Lie group theory, ${ }_{w}^{o b j} \boldsymbol{T}$ is an element of the special Euclidean group $\mathrm{SE}(3)$, while the matrix containing the coordinates of $\boldsymbol{\omega}$ and $\boldsymbol{v}$ is an element of the corresponding Lie algebra $\mathrm{se}(3)$.

\subsection{Screw axis invariants \\ 3.2.1. Time-based descriptor}

The screw axis invariants are the first shape descriptor discussed here, first introduced in [16]. Their definition is based on Chasles' theorem which states that the instantaneous motion of a rigid body can be represented by a rotational and translational velocity along some axis in space, referred to as the Instantaneous Screw Axis (ISA). This theorem is used to describe rigid-body motion trajectories by six invariant functions $\omega_{1}, \omega_{2}, \omega_{3}, v_{1}, v_{2}$, and $v_{3}$. The first two invariants $\omega_{1}$ and $v_{1}$ are the magnitudes of the rotational and translational velocity of the rigid body along the ISA (see Figure 3a). The other invariants model the motion of the ISA with respect to itself in a local moving frame $\{i s a\}$ attached to the ISA. This frame is entirely defined by the geometry of the motion. The first axis ${ }^{i s a} e_{1}$ lies in the direction of the ISA. The second axis ${ }^{i s a} e_{2}$ lies along the common normal between two successive ISAs. The third axis ${ }^{i s a} \boldsymbol{e}_{3}$ follows from the first two because of orthogonality. Two invariants $\omega_{2}$ and $v_{2}$ are defined as the instantaneous rotation and translation of $\{i s a\}$ along the second axis ${ }^{i s a} \boldsymbol{e}_{2}$ (Figure $3 b$ ). The final two invariants $\omega_{3}$ and $v_{3}$ are the instantaneous rotation and translation of the frame along the first axis ${ }^{i s a} e_{1}$ (Figure $3 c)$. Analytical formulas for calculating the ISA invariants from the rigid-body velocity ( $\boldsymbol{\omega}$ and $\boldsymbol{v}$ ) and their first- and second-order time-derivatives are given in [16]. A noise- 


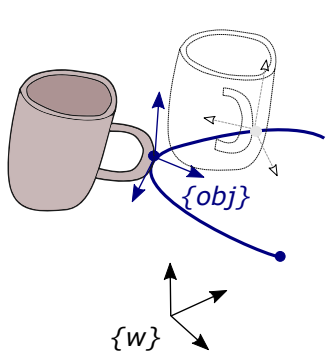

(a)

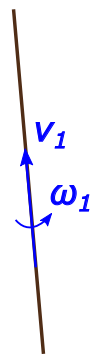

Figure 3: The shape of the six-DOF

motion trajectory of a rigid body can be characterized in a coordinate-free way by six screw axis invariants. These invariants are defined and expressed in a moving frame $\{i s a\}$, that is attached to the instantaneous screw axis. (a) Two invariants $\omega_{1}$ and $v_{1}$ describe the motion of the object around the screw axis. (b) Two invariants $\omega_{2}$ and $v_{2}$ describe the motion of the screw axis along the common normal $\mathbf{e}_{y}$ between successive screw axes. (c) Two invariants $\omega_{3}$ and $v_{3}$ describe the motion of the screw axis frame along $\mathbf{e}_{x}$.

and singularity-robust numerical calculation method can be found in [43].

Denoting the pose of the moving frame $\{i s a\}$ with respect to $\{w\}$ using ${ }_{w}^{i s a} \boldsymbol{T}$, then the time-derivative of this pose ${ }_{w}^{i s a} \boldsymbol{T}^{\prime}$ is written as a function of $\omega_{2}, \omega_{3}, v_{2}$, and $v_{3}$ as follows:

$$
{ }_{w}^{i s a} \boldsymbol{T}^{\prime}={ }_{w}^{i s a} \boldsymbol{T}\left[\begin{array}{cccc}
0 & 0 & \omega_{2} & v_{3} \\
0 & 0 & -\omega_{3} & v_{2} \\
-\omega_{2} & \omega_{3} & 0 & 0 \\
0 & 0 & 0 & 0
\end{array}\right] .
$$

This expression defines the motion of the moving frame as a function of the invariants. Comparing this expression with (1), it becomes clear that the invariants correspond to elements of the rotational and translational velocity vector of $\{i s a\}$ with respect to $\{w\}$. The time-derivative of the rigidbody trajectory ${ }_{w}^{o b j} T^{\prime}$ is found using the two remaining invariants $\omega_{1}$ and $v_{1}$ as follows:

$$
{ }_{w}^{o b j} \boldsymbol{T}^{\prime}={ }_{w}^{i s a} \boldsymbol{T}\left[\begin{array}{cccc}
0 & 0 & 0 & v_{1} \\
0 & 0 & -\omega_{1} & 0 \\
0 & \omega_{1} & 0 & 0 \\
0 & 0 & 0 & 0
\end{array}\right]{ }^{o b j} \boldsymbol{T} .
$$

The structure of (3) is similar to (2), except for the last term ${ }_{\text {isa }}^{\text {obj }} \boldsymbol{T}$, which denotes the pose of the object frame with respect to the ISA frame. This term is necessary for transferring the effect of the invariants, which are defined along the ISA, to the object.

We call (2) and (3) the generative equations of the shape descriptor. They allow reconstruction of the original trajectory ${ }_{w}^{o b j} \boldsymbol{T}(t)$ from the screw axis invariants, given the initial poses of the screw axis frame ${ }_{w}^{i s a} \boldsymbol{T}\left(t_{0}\right)$ and the object frame ${ }_{w}^{o b j} \boldsymbol{T}\left(t_{0}\right)$. A numerical integration procedure for doing this reconstruction will be provided later in Section 4.4.

\subsubsection{Geometric descriptor}

In [16], it is shown that the six screw axis invariants are independent of the chosen world reference frame $\{w\}$ and object frame $\{o b j\}$ during the recording of the trajectory coordinates. However, the invariants are still time-dependent,

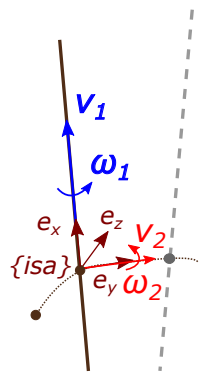

(b)

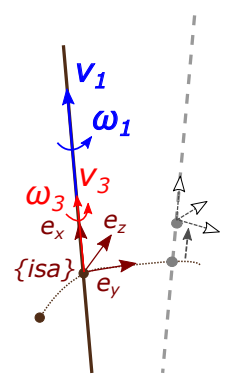

(c) meaning a change in duration or velocity profile would still affect the invariants. To eliminate this dependency, the invariants are expressed as a function of a geometric progress parameter along the trajectory. To have a progress parameter that works in the special cases of pure translation and pure rotation, the approach of [16] is followed. The progress parameter $\xi(t)$ is defined through the rate of advancement $\dot{\xi}(t)$, which is chosen as a weighted combination between progress in translation and progress in rotation along the ISA:

$$
\xi(t)=\int_{t_{0}}^{t} \dot{\xi}(\bar{t}) d \bar{t}, \quad \text { with } \dot{\xi}(t)=w \frac{\left|\omega_{1}\right|}{\Theta}+(1-w) \frac{\left|v_{1}\right|}{L},
$$

where $L$ and $\Theta$ are needed as scaling factors so that $\xi(t)$ goes from 0 to 1 :

$$
L=\int_{t_{0}}^{t_{f}}\left|v_{1}\right| d t ; \Theta=\int_{t_{0}}^{t_{f}}\left|\omega_{1}\right| d t .
$$

The weighting factor $w$ is arbitrarily chosen between 0 and 1. Every invariant $\omega_{k}$ or $v_{k}$ is now re-parameterized, while the velocity is factored out by dividing with $\dot{\xi}$ :

$$
\Omega_{k}(\xi) \stackrel{\Delta}{=} \frac{\omega_{k}(t(\xi))}{\dot{\xi}(t(\xi))} ; \quad V_{k}(\xi) \stackrel{\Delta}{=} \frac{v_{k}(t(\xi))}{\dot{\xi}(t(\xi))}, k=1 \ldots 3 .
$$

The generative equations (2) and (3) retain the same expression when the time-based invariants $\omega_{k}$ and $v_{k}$ are replaced by the geometric invariants $\Omega_{k}$ and $V_{k}$, only now everything (including the derivative) is expressed as a function of $\xi$ instead of $t$. Reconstruction of the original trajectory coordinates now also requires the progress parameter $\xi(t)$, i.e. the motion profile.

\subsection{Extended Frenet-Serret invariants}

The extended Frenet-Serret invariants are the second shape descriptor discussed here, first introduced in [41, 38]. The main difference with the screw axis invariants is that rotation and translation are described separately, instead of being coupled through the screw axis. For the translation, the motion of a chosen reference point on the body is 


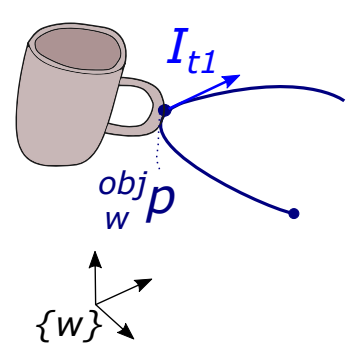

(a)

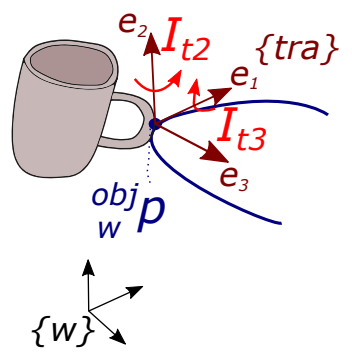

(b)
Figure 4: The three-DOF translation of a point ${ }_{w}^{o b j} \mathbf{p}$ on the rigid body can be characterized in a coordinate-free way by three translational Frenet-Serret invariants, defined and expressed in a moving frame $\{t r a\}$. (a) One invariant $I_{t 1}$ is the translation of the object along the tangent to the curve. (b) Two invariants $I_{t 2}$ and $I_{t 3}$ describe the rotation of $\{$ tra $\}$ itself.

taken, meaning the descriptor would change if another point on the body is chosen. Though this invariant property is now lost with respect to the screw axis invariants, the extended Frenet-Serret invariants are often simpler to use in practice. Below, the translational and rotational parts are explained separately. We immediately go to the geometric descriptor, though a time-based descriptor also exists.

\subsubsection{Object translation}

Suppose the translation of the rigid-body trajectory is given by the trajectory coordinates ${ }_{w}^{o b j} \boldsymbol{p}(s)$ of a chosen reference point on the object. The coordinates are expressed as a function of the normalized arc length $s$ along the trajectory. At the start of the motion $s=0$, while at the end of the motion $s=1$. The invariants for translation $\left(I_{t 1}, I_{t 2}\right.$ and $\left.I_{t 3}\right)$ are defined and expressed in a moving frame, referred to as the translational Frenet-Serret frame $\{$ tra $\}$. This frame is an orientation frame [15], i.e its position in space does not matter, but for visualization purposes the origin is often set to coincide with the point's location, as in Figure 4b. The first axis of this frame is the tangent to the point trajectory, the second axis lies in the direction along which the tangent is instantaneously rotating, and the third axis follows from the first two axes through orthogonality. The first invariant $I_{t 1}$ corresponds to the progress in translation of the point along the tangent. The second invariant $I_{t 2}$ is the instantaneous rotation along the second axis of $\{$ tra $a$ that reorients the tangent. And the third invariant $I_{t 3}$ is the necessary rotation along the first axis to reorient the second axis. $I_{t 2}$ and $I_{t 3}$ are closely related to the curvature and torsion along the trajectory. ([43] provides a robust numerical procedure for calculating $I_{t 2}$ and $I_{t 3}$ from the trajectory coordinates.) Denoting the orientation of the moving frame $\{$ tra $\}$ using the $3 \times 3$ rotation matrix ${ }_{w}^{t r a} \boldsymbol{R}$, the change in orientation ${ }_{w}^{\operatorname{tra}} \boldsymbol{R}^{\prime}$ is defined as a function of $I_{t 2}$ and $I_{t 3}$ as follows:

$$
{ }_{w}^{t r a} \boldsymbol{R}^{\prime}={ }_{w}^{\operatorname{tra}} \boldsymbol{R}\left[\begin{array}{ccc}
0 & 0 & I_{t 2} \\
0 & 0 & -I_{t 3} \\
-I_{t 2} & I_{t 3} & 0
\end{array}\right] .
$$

These equations are also known as the Frenet-Serret differential equations [25], though note we use a different order of the axes to be consistent with the screw axis invariants. The change in point coordinates ${ }_{w}^{o b j} \boldsymbol{p}^{\prime}(s)$ is found using the remaining invariant $I_{t 1}$ :

$$
{ }_{w}^{o b j} \boldsymbol{p}^{\prime}={ }_{w}^{t r a} \boldsymbol{R}\left(\begin{array}{c}
I_{t 1} \\
0 \\
0
\end{array}\right),
$$

where $I_{t 1}$ can be show to be a constant value equal to the total trajectory length $L$, signifying a constant progression in translation. These two equations allow reconstruction of the point trajectory from the invariants $I_{t 1}, I_{t 2}$ and $I_{t 3}$, given the initial location of the point ${ }_{w}^{o b j} \boldsymbol{p}(0)$ and the initial translational Frenet-Serret frame ${ }_{w}^{\operatorname{tra}} \boldsymbol{R}(0)$, which signifies the initial traveling direction.

\subsubsection{Object rotation}

The orientation of the rigid-body trajectory, given by the $3 \times 3$ rotation matrix ${ }_{w}^{o b j} \boldsymbol{R}(\theta)$, is handled analogously. ${ }_{w}^{o b j} \boldsymbol{R}(\theta)$ is expressed as a function of the normalized rotated angle $\theta$, which is the integral of the rotational velocity along the trajectory: $\theta(t)=\int_{t_{0}}^{t} \frac{\|\omega(\bar{t})\|}{\Theta} d \bar{t}$, with $\Theta$ the total rotated angle so that at the start of the motion, $\theta=0$, while at the end of the motion, $\theta=1$.

The three invariants for rotation $\left(I_{r 1}, I_{r 2}\right.$ and $\left.I_{r 3}\right)$ are again defined using a moving frame, referred to as the rotational Frenet-Serret frame $\{$ rot $\}$. The first axis of this frame (the tangent) lies in the direction of $\omega$, the second axis lies in the direction along which the first axis is instantaneously rotating, and the third axis follows from the first two axes through orthogonality. The first invariant $I_{r 1}$ is oriented along the first axis of $\{r o t\}$ and is a measure of the progress in rotation of the object. The second and third invariants, $I_{r 2}$ and $I_{r 3}$, are again the rotational velocities of the moving frame $\{r o t\}$ itself, and are oriented along the second and first axis of $\{r o t\}$, respectively. Denoting the orientation of $\{r o t\}$ using ${ }_{w}^{r o t} \boldsymbol{R}$, the change in orientation ${ }_{w}^{r o t} \boldsymbol{R}^{\prime}$ is then defined as:

$$
{ }_{w}^{r o t} \boldsymbol{R}^{\prime}={ }_{w}^{r o t} \boldsymbol{R}\left[\begin{array}{ccc}
0 & 0 & I_{r 2} \\
0 & 0 & -I_{r 3} \\
-I_{r 2} & I_{r 3} & 0
\end{array}\right] .
$$

The change in the orientation of the rigid body, denoted as ${ }_{w}^{o b j} \boldsymbol{R}^{\prime}$, is found using the remaining invariant $I_{r 1}$ :

$$
{ }_{w}^{o b j} \boldsymbol{R}^{\prime}={ }_{w}^{r o t} \boldsymbol{R}\left[\begin{array}{ccc}
0 & 0 & 0 \\
0 & 0 & -I_{r 1} \\
0 & I_{r 1} & 0
\end{array}\right]{ }_{r o t}{ }^{o b j} \boldsymbol{R},
$$

where $I_{r 1}$ can be shown to be equal to $\Theta$, signifying a constant progression in object rotation.

\subsection{General form generative equations}

The generative equations for reconstructing the object trajectory from the screw axis invariants (2)-(3) and the extended Frenet-Serret invariants (5)-(8) share some similarities. Both shape descriptors use moving frames in which 
Table 1

Overview of generative equations for reconstructing the object trajectory from the coordinate-free shape descriptor for a general shape descriptor, the screw axis invariants, and the extended Frenet-Serret invariants.

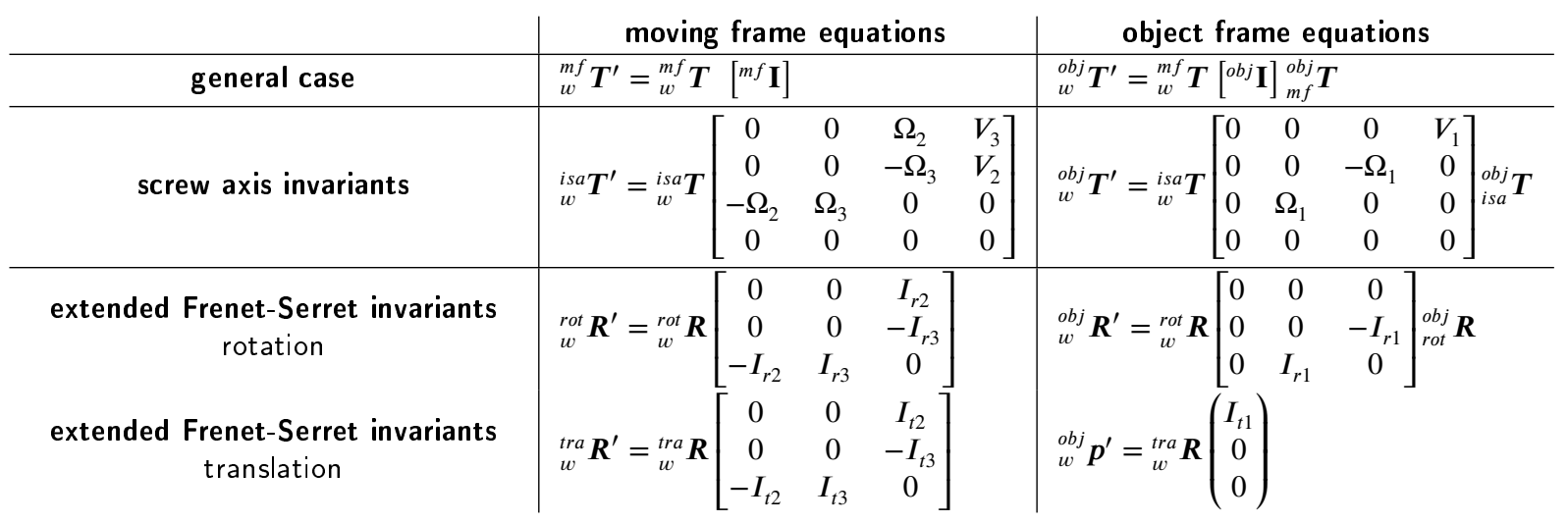

invariants are defined. Some of these invariants describe the motion of the moving frame while other invariants describe the motion of the object itself. This subsection aims to formulate a general expression of these generative equations, valid for any shape descriptor that relies on a moving frame approach.

\subsubsection{Moving frame equations}

The moving frame will be generally referred to as $\{m f\}$ here. The pose of $\{m f\}$ with respect to $\{w\}$ is given by ${ }_{w}^{m f} \boldsymbol{T}$. We refer to the invariants of the shape descriptor that describe its rotation and translation as the moving frame invariants. These invariants are generally expressed using the $6 \times 1$ twist vector ${ }^{m f} \mathbf{I}=\left(\begin{array}{c}m f \\ { }^{m f} \mathbf{V}\end{array}\right)$, where ${ }^{m f} \boldsymbol{\Omega}$ represents the change in the moving frame's orientation and ${ }^{m f} \mathbf{V}$ represents the change in translation. When parameterized in time, these vectors correspond to the rotational and translational velocity of the moving frame. The coordinates of both vectors are expressed in the moving frame $\{m f\}$.

Given a general progress parameter $\tau$ along the trajectory, which may be the time $t$ or a geometric progress parameter such as the arc length $s$ or rotated angle $\theta$, the derivative of the pose with respect to $\tau$ as a function of the invariants ${ }^{m f} \mathbf{I}$ can be noted compactly as:

$$
{ }_{w}^{m f} \boldsymbol{T}^{\prime}(\tau)={ }_{w}^{m f} \boldsymbol{T}(\tau)\left[{ }^{m f} \mathbf{I}(\tau)\right]
$$

The notation [·] applied to the twist vector ${ }^{m f} \mathbf{I}$ constructs the following matrix from the vector:

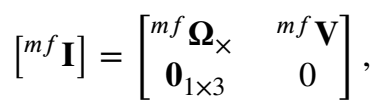

with ${ }^{m f} \boldsymbol{\Omega}_{\times}$the skew-symmetric matrix constructed from ${ }^{m f} \boldsymbol{\Omega}$. From (9), the moving frame equations for the specific shape descriptors can be derived. Table 1 provides an overview. E.g. for the translational Frenet-Serret invariants, the moving frame $\{$ tra $\}$ is an orientation frame, so ${ }_{w}^{m f} \boldsymbol{T}$ reduces to ${ }_{w}^{\operatorname{tra}} \boldsymbol{R}$. The moving frame invariants ${ }^{m f} \mathbf{I}$ are likewise reduced to ${ }^{m f} \boldsymbol{\Omega}$, which is set equal to $\left(\begin{array}{lll}I_{t 3} & I_{t 2} & 0\end{array}\right)^{T}$. Substituting this into (9), we again obtain expression (5).

\subsubsection{Object frame equations}

We refer to the invariants of the shape descriptor that describe the rotation and translation of the rigid object as the object frame invariants. They are also generally expressed using a twist vector ${ }^{o b j} \mathbf{I}=\left(\begin{array}{c}{ }^{o b j} \boldsymbol{\Omega} \\ { }^{o b j} \mathbf{V}\end{array}\right)$, where the vector ${ }^{o b j} \mathbf{\Omega}$ expresses changes in the object's rotation and the vector ${ }^{o b j} \mathbf{V}$ changes in the object's translation. The coordinates of both vectors are expressed in $\{m f\}$, just like the moving frame invariants. The derivative of the object's pose with respect to $\tau$ can be noted compactly as:

$$
{ }_{w}^{o b j} \boldsymbol{T}^{\prime}(\tau)={ }_{w}^{m f} \boldsymbol{T}(\tau)\left[{ }^{o b j} \mathbf{I}(\tau)\right]{ }_{m f}^{o b j} \boldsymbol{T}(\tau)
$$

where the latter term ${ }_{m f}^{o b j} \boldsymbol{T}$ refers to the pose of the object frame $\{o b j\}$ with respect to the moving frame $\{m f\}$, necessary for relating changes expressed in the moving frame to changes on the object frame. Table 1 lists the object frame equations for the different shape descriptors. E.g. for the translational Frenet-Serret invariants, the moving frame ${ }_{w}^{m f} \boldsymbol{T}$ reduces to ${ }_{w}^{t r a} \boldsymbol{R}$ and the object frame ${ }_{w}^{o b j} \boldsymbol{T}$ reduces to only the translation ${ }_{w}^{o b j}$ p. The object invariants ${ }^{o b j} \mathbf{I}$ reduce to ${ }^{m f} \boldsymbol{V}$, which is set equal to $\left(\begin{array}{lll}I_{t 1} & 0 & 0\end{array}\right)^{T}$. Substituting this into (10) and extracting only the translation yields equation (6) again.

The main advantage of having defined the generic generative equations (9)-(10) is that the motion generalization algorithm of Section 4 is valid for all shape descriptors using a moving frame approach.

\section{Trajectory generalization starting from shape descriptor of demonstration}

Given the shape descriptor of the demonstrated trajectory, new trajectories are generated using constraint-based 
optimization, taking new task requirements into account. First, the general optimization problem is formulated, followed by some remarks for specific shape descriptors. Then, additional path constraints (robot workspace limits and obstacle avoidance) are included. Finally, details are given on the implementation and solution of the optimization problem.

\subsection{Generalization using constrained optimization}

The generative equations (9)-(10) for reconstructing the object trajectory from the general shape descriptor are the starting point for formulating the optimization problem. The optimization problem is structured as an optimal control problem, which is a special class of optimization problem that tries to find an optimal choice of control inputs to drive the states of a system to a desired result. It is specified by an objective function and a set of constraints on the states and controls. The relation between states and controls is given by a dynamical system $\dot{\boldsymbol{x}}(\tau)=\boldsymbol{f}(\boldsymbol{x}, \boldsymbol{u}, \tau)$ which is added in the constraints, specifying the evolution of the states $x$ as a function of the control inputs $\boldsymbol{u} . \tau$ is the progress variable in which $\boldsymbol{x}$ and $\boldsymbol{u}$ are parameterized.

For our problem, the controls $\boldsymbol{u}$ correspond to the object and moving frame invariants of the shape descriptor $\boldsymbol{u}=$ $\left({ }^{o b j} \mathbf{I},{ }^{m f} \mathbf{I}\right)$. The system states consist of the pose of the object and moving frames: $\boldsymbol{x}=\left({ }^{o b j} \boldsymbol{T},{ }^{m f} \boldsymbol{T}\right)$. It becomes clear then that the dynamical system $\dot{x}(\tau)=\boldsymbol{f}(\boldsymbol{x}, \boldsymbol{u}, \tau)$ in the optimal control problem directly corresponds to the generative equations (9)-(10) that were detailed for specific shape descriptors in Table 1.

The optimal control problem is formulated as follows in the general case:

$$
\begin{aligned}
& \underset{\substack{{ }^{o b j} \boldsymbol{T}(\cdot){ }^{o b j} \mathbf{I}(\cdot), w f \\
w}(\cdot),{ }^{m f} \mathbf{I}(\cdot)}{\operatorname{minimize}} \int_{0}^{1}\left\|\left(\begin{array}{c}
{ }^{o b j} \mathbf{I}(\tau)-{ }^{o b j} \mathbf{I}^{\text {demo }}(\tau) \\
{ }^{m f} \mathbf{I}(\tau)-{ }^{m f} \mathbf{I}^{\text {demo }}(\tau)
\end{array}\right)\right\|_{W}^{2} \mathrm{~d} \tau \\
& \text { s.t. } \quad{ }_{w}^{m f} \boldsymbol{T}^{\prime}(\tau)={ }_{w}^{m f} \boldsymbol{T}(\tau)\left[{ }^{m f} \mathbf{I}(\tau)\right] \\
& \left.{ }_{w}^{o b j} \boldsymbol{T}^{\prime}(\tau)={ }_{w}^{m f} \boldsymbol{T}(\tau){ }^{o b j} \mathbf{I}(\tau)\right]_{m f}^{o b j} \boldsymbol{T}(\tau) \\
& \boldsymbol{r}\left({ }_{w}^{o b j} \boldsymbol{T}(j),{ }_{w}^{m f} \boldsymbol{T}(j)\right)=\mathbf{0} \quad \text { for } j=\{0,1\} \\
& \boldsymbol{h}\left({ }_{w}^{o b j} \boldsymbol{T}(\tau),{ }_{w}^{m f} \boldsymbol{T}(\tau)\right) \geq \mathbf{0} \text { for } \tau \in[0,1] .
\end{aligned}
$$

The objective function and constraints are described in more detail below.

Objective function (11a) contains the error between the invariants in the shape descriptor of the generated trajectory $\left({ }^{o b j} \mathbf{I},{ }^{m f} \mathbf{I}\right)$ with respect to the shape descriptor of the demonstrated trajectory $\left({ }^{o b j} \mathbf{I}^{\mathrm{demo} o},{ }^{m f} \mathbf{I}^{\text {demo }}\right.$ ). This error is non-zero if the task constraints (11d)-(11e) differ from the original demonstration. A constant diagonal weighting matrix $W$ weights the relative importance of the invariants and thus controls how the error is distributed. Alternatively, a varying weighting matrix $W(\tau)$ can be used to hold statistical information if multiple demonstrations are available, where a lower variance between invariants corresponds to a higher weight, so that they are more preserved in the generated trajectory.

State dynamics equations (11b)-(11c) are the generative equations that relate the invariant functions of the shape descriptor to the generated trajectory (see Section 3.4).

Boundary constraints (11d) specify the initial and final position and orientation of the object ${ }_{w}^{o b j} \boldsymbol{T}$ and of the moving frame ${ }_{w}^{m f} \boldsymbol{T}$. The significance of constraining ${ }_{w}^{m f} \boldsymbol{T}$ is that it allows to specify the initial and final direction in which the object is moving. If these directions are irrelevant, the corresponding constraints may be omitted. While in (11d) these constraints are expressed as hard constraints, it is also possible to formulate them as soft constraints using a slack variable in the objective function. This is useful, for example, if the desired pose is uncertain. The uncertainty is then expressed through an appropriate weighting factor.

Additional path constraints (11e) are optionally included. For example, to avoid obstacles along the path or to take the workspace limits of the robot platform into account. These additional constraints are further elaborated in Section 4.3.

\subsection{Remarks for specific shape descriptors}

To apply the general formulation to the specific shape descriptors, the following aspects need to be considered.

\subsubsection{Extended Frenet-Serret invariants (eFSI)}

The eFSI decouple the object's translation and rotation by formulating two moving frames with their respective invariants. In the optimal control problem, the dynamics equations thus consist of two equations (5)-(6) for the object's position and two equations (7)-(8) for the object's orientation. The invariants for translation and rotation are also considered separately in the objective function. Note also that the underlying meaning of $\tau$ differs for translation and rotation. For the translation, $\tau$ refers to the arc length $s$, the progress in translation, while for rotation, $\tau$ refers to the rotated angle $\theta$, the progress in rotation. Section 3.3 mentions that the first translational invariant $I_{t 1}(s)$ is constant and equal to the total length $L$. To ensure this relation holds for the generated trajectory, an additional constraint is introduced: $I_{t 1}(s)=L$, for $s \in[0,1] . L$ is considered an unknown parameter to be found during optimization. A similar constraint is introduced for the rotational invariant: $I_{r 1}(\theta)=$ $\Theta$, for $\theta \in[0,1]$.

\subsubsection{Screw axis invariants (SAI)}

The SAI couple translation and rotation through the screw axis. This coupling is also visible in the definition of the geometric progress variable $\xi(t)$ in (4). For the geometric SAI, this relation becomes the following after division by the velocity $\dot{\xi}(t): 1=w \frac{\left|\Omega_{1}(\xi)\right|}{\Theta}+(1-w) \frac{\left|V_{1}(\xi)\right|}{L}$, for $\xi \in[0,1]$. Similarly to the eFSI, this relation is added as a constraint, with $L$ and $\Theta$ as unknown parameters. 


\subsection{Including additional path constraints}

One of the major advantages of generating trajectories using constraint-based optimization is that additional constraints are easy to include. We further explain two types of additional constraints: robot workspace limits and obstacle avoidance.

Robot workspace limits are often only considered in a secondary inverse kinematics or inverse dynamics step, after the desired trajectory has already been calculated in Cartesian space with a different method. However, this does not guarantee that the generated trajectory is inside the workspace of the robot. When trajectory generation is done using constrained optimization, the robot workspace can be taken into account directly by including the kinematic model as part of the constraints:

$$
{ }_{w}^{o b j} \boldsymbol{T}(\tau)=\boldsymbol{f}_{k i n}(\boldsymbol{q}(\tau)) \text {, with } \boldsymbol{q}_{l} \leq \boldsymbol{q}(\tau) \leq \boldsymbol{q}_{u},
$$

in which $\boldsymbol{q}$ is the vector of joint angles, $\boldsymbol{q}_{l}$ and $\boldsymbol{q}_{u}$ are the lower and upper joint angle limits, and $\boldsymbol{f}_{\text {kin }}$ is the forward kinematic model of the robot including the object attached to the end-effector. The approach is readily extended to dynamic robot models, hence with constraints expressed on the joint torques.

Obstacle avoidance is another important aspect of trajectory generation. Collisions need to be avoided between the robot and its environment and also between segments of the robot itself. Often the problem is simplified by assuming convex objects. Including collision avoidance in optimization problems is achievable in multiple ways. One approach models obstacles as convex polyhedra, between which the GJK algorithm [19] easily calculates the distance. This distance measurement between object and obstacle is then added as an inequality constraint to ensure it remains above a certain safe threshold, as for example was done in [37]. Another approach is to use the separating hyperplane theorem between convex sets in order to constrain convex objects to remain on their respective side of the hyperplane [10]. Both approaches are fully compatible with the formulated optimization problem in (11a)-(11e).

\subsection{Discretization and numerical solution}

The continuous optimal control problem (OCP) is first discretized and afterwards solved numerically.

Discretization: The OCP is discretized with a direct multiple shooting method [17]. Both states and controls are discretized by partitioning the progress parameter $\tau$ in $N$ intervals, yielding $x_{k}$ and $u_{k}$ for $k=\{0,1, \ldots, N\}$. The controls $u_{k}$ are considered piecewise constant over these intervals. For the state dynamics $\dot{x}(\tau)=\boldsymbol{f}(\boldsymbol{x}, \boldsymbol{u}, \tau)$, an integrator function $\boldsymbol{F}$ is defined so that the dynamics equations are transformed into the following equality constraints: $\boldsymbol{x}_{k+1}-\boldsymbol{F}\left(\boldsymbol{x}_{k}, \boldsymbol{u}_{k}\right)=0$. To correctly integrate the pose dynamics in (9) and (10), a geometric integrator is needed to ensure that the properties of the special Euclidean group $\mathrm{SE}(3)$ are preserved. Pose dynamics that are in the form of (1):

$$
\boldsymbol{T}^{\prime}(\tau)=\boldsymbol{T}(\tau)\left[\begin{array}{cc}
{[\boldsymbol{\omega}(\tau)]_{\times}} & \boldsymbol{v}(\tau) \\
\mathbf{0}_{1 \times 3} & 0
\end{array}\right],
$$

are exactly integrated using exponential mapping [29], if $\boldsymbol{\omega}_{k}$ and $\boldsymbol{v}_{k}$ are constant over the integration interval $\Delta \tau$ :

$$
\boldsymbol{T}_{k+1}=\boldsymbol{T}_{k} \exp \left(\left[\begin{array}{cc}
{\left[\boldsymbol{\omega}_{k}\right]_{\times}} & \boldsymbol{v}_{k} \\
\mathbf{0}_{1 \times 3} & 0
\end{array}\right] \Delta \tau\right),
$$

This property is used to define the integrator for (11b)-(11c). The result of this discretization process on the optimal control problem of (11a)-(11e) is the following nonlinear optimization problem (NLP):

$$
\begin{aligned}
& \underset{\substack{{ }^{o b j} \boldsymbol{T}_{(\cdot)}{ }_{m f}{ }^{o b j} \mathbf{I}_{(\cdot)},{ }_{w} \boldsymbol{T}_{(\cdot)},{ }^{m f} \mathbf{I}_{(\cdot)}}}{\operatorname{minimize}} \quad \sum_{k=0}^{N}\left\|\left(\begin{array}{c}
{ }^{o b j} \mathbf{I}_{k}-{ }^{o b j} \mathbf{I}_{k}^{\text {demo }} \\
{ }^{m f} \mathbf{I}_{k}-{ }^{m f} \mathbf{I}_{k}^{\text {demo }}
\end{array}\right)\right\|_{W} \\
& \text { s.t. } \quad{ }_{w}^{m f} \boldsymbol{T}_{k+1}-{ }_{w}^{m f} \boldsymbol{T}_{k} \exp \left(\left[{ }^{m f} \mathbf{I}_{k}\right] \Delta \tau\right)=0 \text {, } \\
& { }_{w}^{o b j} \boldsymbol{T}_{k+1}-{ }_{w}^{m f} \boldsymbol{T}_{k} \exp \left(\left[{ }^{o b j} \mathbf{I}_{k}\right] \Delta \tau\right){ }_{m f}^{o b j} \boldsymbol{T}_{k}=0, \\
& \text { for } k=0, \ldots, N-1 \\
& \boldsymbol{r}\left({ }_{w}^{o b j} \boldsymbol{T}_{j},{ }_{w}^{m f} \boldsymbol{T}_{j}\right)=\mathbf{0}, \quad \text { for } j=\{0, N\} \\
& \boldsymbol{h}\left({ }^{o b j} \boldsymbol{T}_{k},{ }_{w}^{m f} \boldsymbol{T}_{k}\right) \geq \mathbf{0}, \quad \text { for } k=0, \ldots, N .
\end{aligned}
$$

Implementation and solution: The open-source software CasADi [4] is used for implementing the discretized optimization problem (15a)-(15e) using symbolic expression graphs that allow efficient numerical evaluation of functions and derivatives. The actual solving is done by the IPOPT numerical solver [44], which uses an interior-point primal-dual Newton method with line-search. The states and controls are initialized with the trajectory and invariants of the demonstration.

The NLP is not convex, hence a global solution is not guaranteed. However, in practice, the local solution is often good enough. If obtaining the global solution is important, the optimization problem can be solved multiple times with different initial values to select the solution with the lowest cost.

\section{Experiments}

The proposed method for generalizing trajectories using coordinate-free shape descriptors is evaluated in multiple ways. First, the trajectory of a single demonstrated motion is shown to be successfully adapted to various new task requirements. Then, a comparison is made between two specific types of shape descriptors: the extended Frenet-Serret Invariants (eFSI) and the Screw Axis Invariants (SAI), which were explained in Section 3. Finally, a comparison is made between the shape descriptor approach for trajectory generalization and two approaches not using shape descriptors: dynamic movement primitives [21] and jerk-accuracy optimization [28]. 

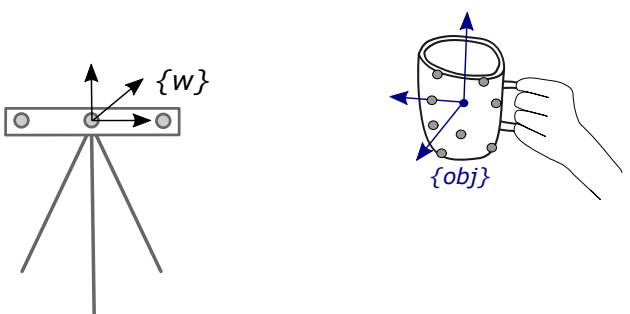

Figure 5: Graphical representation of measurement setup and definition of reference frames during recording.

\subsection{From measurements to shape descriptor}

The rigid-body trajectory of a human-manipulated object (coffee mug) is measured by attaching markers to the object and tracking these markers using a Krypton K600 vision system from NIKON Metrology (as depicted in Figure 5). The vision system consists of three CCD cameras that determine the position of the active led marker through triangulation, at a rate of $50 \mathrm{~Hz}$ with an expected accuracy of $60 \mu \mathrm{m}$ [1]. The reference frame of the measurements $\{w\}$ corresponds to the reference frame of the camera. From these marker trajectories, the pose of the object $\{o b j\}$ can be calculated, where the reference point for the translation of the object is chosen as the average position of all the markers.

The eFSI and SAI shape descriptors of Section 3 are then calculated from the measured trajectory coordinates. Classical analytical formulas may suffer from noise sensitivity and the influence of singularities (where invariants become undefined). Therefore, we use the numerical approach of [43], which reformulates the shape descriptor calculation as an optimal estimation problem that directly finds the shape descriptor that reconstructs the given measured trajectory.

\subsection{Generalization to new task constraints}

The generalization and adaptation capabilities are illustrated for the trajectory of a demonstrated pouring motion. Starting from this single demonstration, the motion trajectory is adapted to different situations by imposing new task constraints in the optimization problem (15a)-(15e).

The geometric eFSI of Section 3.3 were chosen as the specific type of shape descriptor here, with equal weight on every invariant. Since for this choice of shape descriptor, the solution of the optimization problem only generates the geometry of the trajectory (the path), a velocity profile needs to be applied to the solution to expresses the object's position and orientation again as a function of time. Here, we chose to re-apply the velocity profile of the original demonstration. However, other methods could just as well be chosen to adjust the timing along the trajectory such as, for example, a minimum-jerk constraint along the given path, as was done in [8]. These types of constraints are easy to include in the optimization problem.

For each of the experiments, the average number of iterations, number of samples $N$, and time per iteration is shown in Table 2, executed on an Intel Core i7-4790 processor.
Table 2

Average number of iterations and time/iteration for the trajectory generalization experiments with new task constraints.

\begin{tabular}{l|c|c|c} 
experiment & samples [N] & iterations & time/iteration [s] \\
\hline New target pose & 65 & 6 & 0.0143 \\
New target pose & 22 & 6 & 0.0108 \\
New initial pose & 65 & 10 & 0.0146 \\
New target approach direction & 65 & 20 & 0.0137 \\
On-line adaptation & 33 & 10 & 0.0125 \\
Avoid obstacle & 65 & 18 & 0.0147 \\
Robot workspace limits & 65 & 41 & 0.0507
\end{tabular}

\subsubsection{New target pose}

To adapt the demonstrated pouring motion to different target poses (e.g. different glasses on the table to pour into), the end constraint ${ }_{w}^{o b j} \boldsymbol{T}_{N}$ on the object's position and orientation is set to the desired value in (15d). Figure 6a shows how the demonstrated trajectory (in blue) is adapted to different chosen target poses (in red), while Figure 6b displays the corresponding changes in the invariants of the shape descriptor. Most of the invariants are nearly coinciding here. Note that $I_{t 1}$ and $I_{r 1}$ have constant values, since they contain the global scale in translation and rotation respectively (see Section 3.3). Since the change in the invariants is small, shape-similarity with the original trajectory is preserved.

\subsubsection{New initial pose}

Figure 7 illustrates how the trajectory is adapted due to changes in the starting position and orientation of the object ${ }_{w}^{o b j} \boldsymbol{T}_{0}$ in (15d). For the chosen initial poses, a deviation in shape is required to satisfy the constraints. For example, for the first generated trajectory in Figure 7, there is an increase in length $I_{t 1}$ and a decrease in overall curvature $I_{t 2}$ to achieve the desired constraint on the initial pose.

\subsubsection{New target approach direction}

Figure 8 shows how the trajectory adapts to changes in the target approach direction, indicated by the large red arrows near the target. As explained in Section 4.1, the target approach direction can be set by constraining the moving frame at the target ${ }_{w}^{m f} \boldsymbol{T}_{N}$ in (15d). In this case, the first axis of the translational Frenet-Serret frame $\{$ tra $\}$ is set so that it lies in the direction of the desired tangent. Large changes in the translational invariants $I_{t 2}$ and $I_{t 3}$, especially at the end, are necessary to adjust the direction.

\subsubsection{On-line adaptation}

While the whole trajectory can be generated at once by solving the optimization problem, it is also possible to generate updated trajectories if there is a change or perturbation in the environment during the motion. Figure 9 shows what happens if, after $50 \%$ of the translation of the original trajectory (indicated by the black dot), the target position is suddenly changed to another position. By requiring in (15d) that the new trajectory starts from the current state (current position ${ }_{w}^{o b j} \boldsymbol{T}_{N / 2}$ and direction ${ }_{w}^{m f} \boldsymbol{T}_{N / 2}$ ), the trajectory is smoothly adapted to the new target location by changes in 


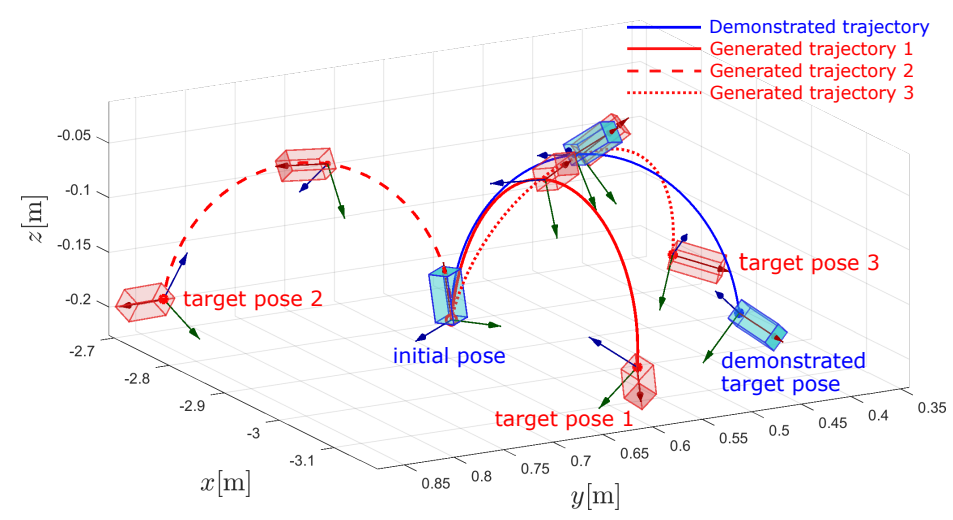

(a) New target poses for the same initial pose
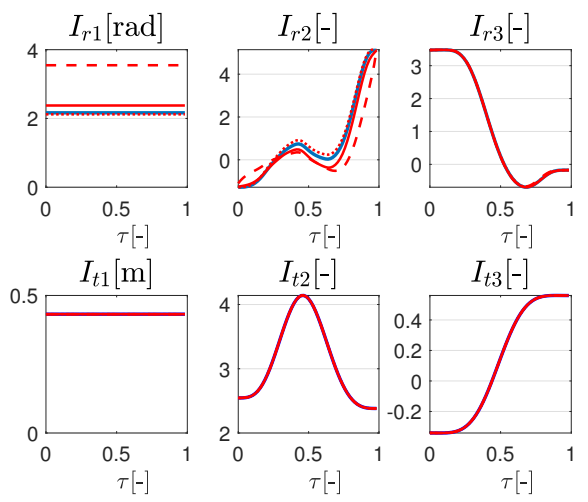

(b) eFSI shape descriptor

Figure 6: Adaptation of the demonstrated pouring motion trajectory (blue) to new target positions and orientations (red). The trajectory is adjusted through corresponding small changes in the extended Frenet-Serret invariant (eFSI) shape descriptor.

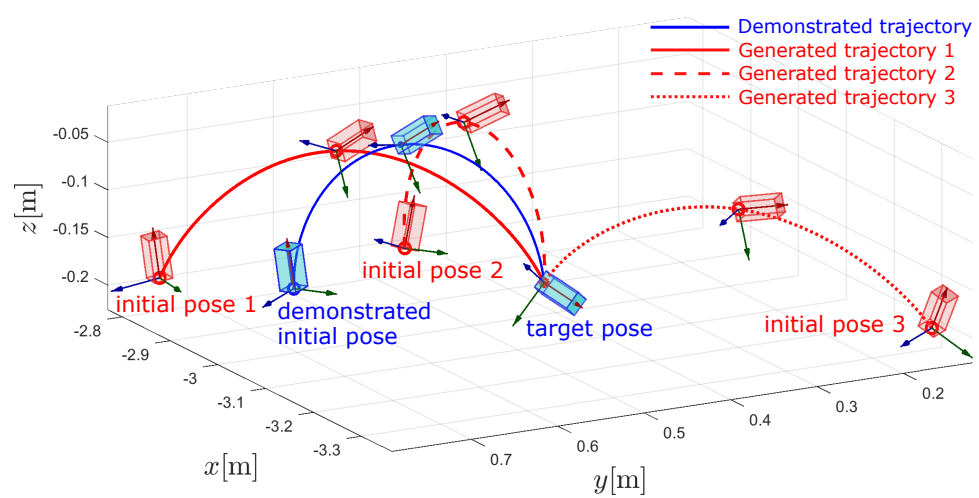

(a) New initial poses for the same target pose

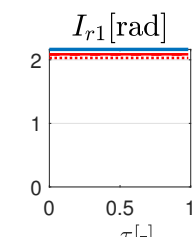

$\tau[-]$
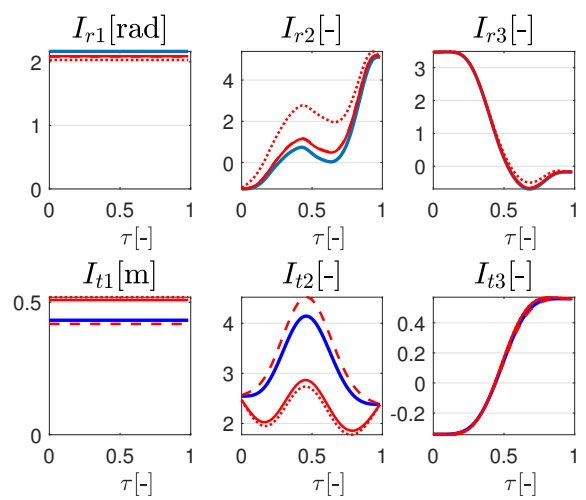

(b) eFSI shape descriptor

Figure 7: Adaptation of the demonstrated pouring motion trajectory (blue) to new initial positions and orientations (red), for the same target pose. Changes in the shape descriptor are required to adjust to the new task requirements.

the translational invariants $I_{t 1}, I_{t 2}$ and $I_{t 3}$, while maintaining second-order continuity in the trajectory.

\subsubsection{Avoid static obstacle}

A static obstacle is added in the additional constraints (11e) of the optimization problem. A simple type of obstacle, a sphere, was added in Figure 10 along the demonstrated trajectory, but as explained in Section 4.3, similar procedures exist for more complex, convex obstacles. Changes in the translational invariants $I_{t 1}, I_{t 2}$ and $I_{t 3}$ of the shape descriptor are now required to avoid the obstacle and keep the same initial and target pose. Still, the new trajectory looks similar to the demonstrated one. Dynamic obstacles can also be included in the optimization if their motion can be estimated over the considered horizon.

\subsubsection{Robot workspace limits}

Figure 11 shows the adaptation of the demonstrated trajectory in two distinct cases where the workspace limits of a 6-DOF robot manipulator are taken into account. The start and end of the trajectory lie inside the workspace, while the middle part lies outside the workspace, thus requiring an adaptation. In Figure 11a, the object's trajectory was calculated first and afterwards the robot joint trajectories are obtained using inverse kinematics in a separate step. The trajectory part outside of the workspace was consequently projected on the workspace boundary, which results in a large distortion of trajectory shape that can also be seen in the invariants of the shape descriptor. In Figure $11 \mathrm{~b}$ however, the robot kinematic model is directly included in constraint (15e), as explained in Section 4.3. The effect is that the whole calculated trajectory lies inside the workspace and that the shape is better preserved.

\subsection{Comparison between shape descriptors}

The constraint-based optimization approach for trajectory generalization is compared for two types of shape descriptors for rigid-body motion: the extended Frenet-Serret Invariants (eFSI) and the Screw Axis Invariants (SAI). The most important difference in invariant properties between 


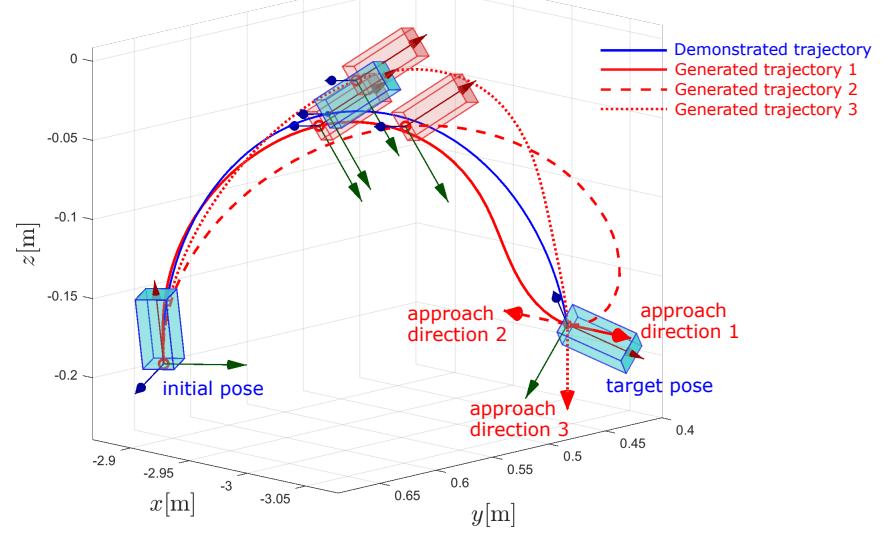

(a) New target approach directions
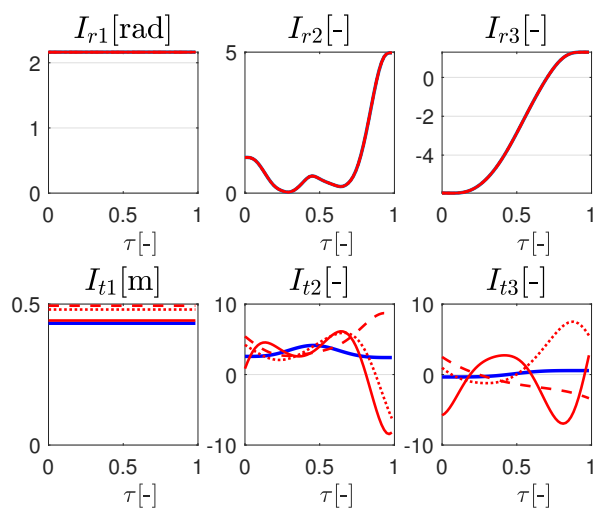

(b) eFSI shape descriptor

Figure 8: Adaptation of the demonstrated pouring motion trajectory (blue) to new target approach directions (red), indicated by the large red arrows near the target pose, while maintaining the original demonstrated target pose.

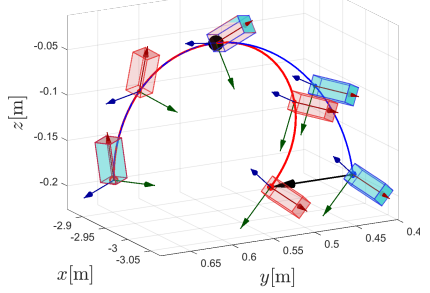

(a) New target pose
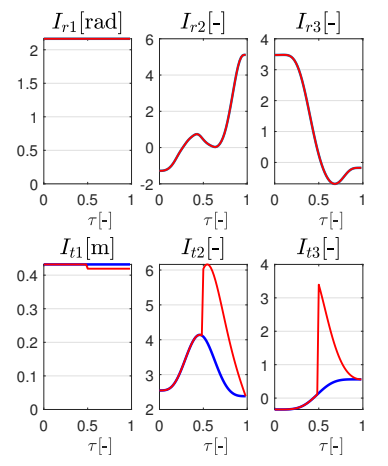

(b) eFSI shape descriptor
Figure 9: Smooth on-line adaptation of the demonstrated pouring trajectory (blue) to a new target position (red) after $50 \%$ translation (black dot) along the original trajectory. The translational invariants deviate after $s=0.5$ to address the change in target position.

eFSI and SAI is the dependency of the eFSI on the choice of reference point ${ }_{w}^{o b j} \boldsymbol{p}$ on the object for the translation. Figure 12 illustrates this property. The goal is to reconstruct the demonstrated trajectory for the same initial and final pose, but now choosing a different reference point (black dot) for the translation. Practically, you could imagine it as holding a different part of the object and wanting the object to execute the exact same trajectory. For the eFSI descriptor, the reconstructed object trajectory and the corresponding invariants are noticeably different. This is because the translational invariants $I_{t 1}, I_{t 2}$ and $I_{t 3}$ were calculated for another reference point during the demonstration. However, if the position of the original reference point is known, the invariants could still be corrected analytically. For the SAI descriptor, no changes in trajectory and invariant space happen since these invariants do not depend on the choice of reference point.

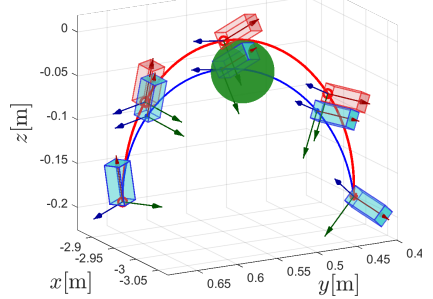

(a) Avoiding spherical obstacle
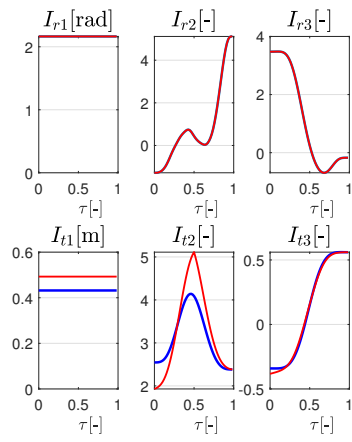

(b) eFSI shape descriptor
Figure 10: Adaptation (red) of the demonstrated pouring motion trajectory (blue) to avoid a static obstacle (green) lying along the original demonstrated trajectory.

\subsection{Comparison with trajectory adaptation methods not using shape descriptors}

The generalization method using coordinate-free shape descriptors is compared with two other methods for trajectory adaptation not using shape descriptors. Again only a single demonstration is considered. Only the translation of the object is investigated, not the rotation, for an easier comparison. For all methods, the initial and target positions are specified, while the velocity and acceleration are considered unconstrained at the start and end. The results are shown in Figure 13.

\subsubsection{Jerk-accuracy optimization}

The idea behind jerk-accuracy optimization [28] is similar to our optimization method using shape descriptors. Instead of minimizing the deviation from the demonstration in invariant space however, the deviation is directly minimized in Cartesian (task) space. This deviation, the mean square error on the position, is referred to as the accuracy cost. The objective function is supplemented with a jerk cost term in 


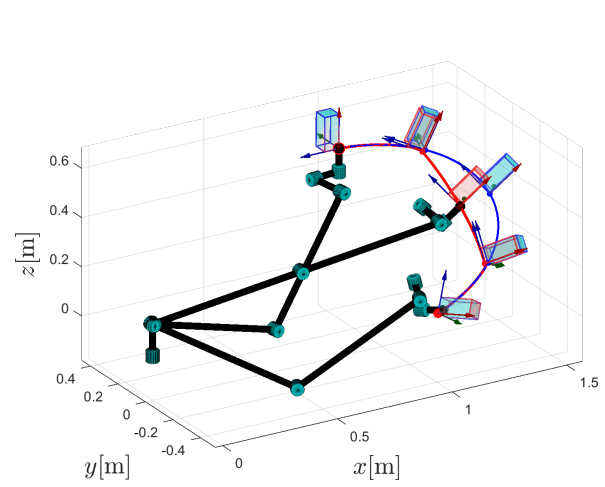

(a) Inverse kinematics in separate step

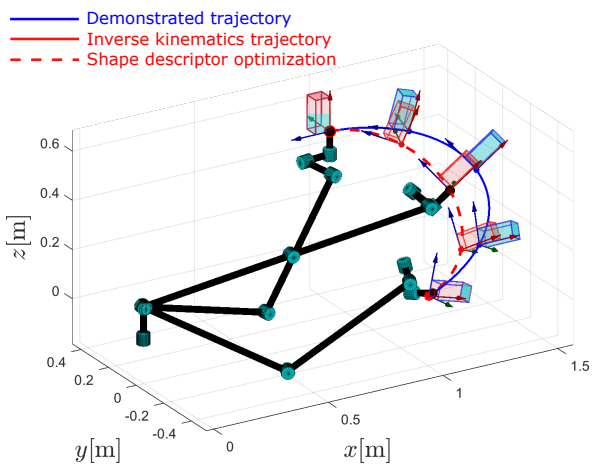

(b) Inclusion of kinematic model in optimization

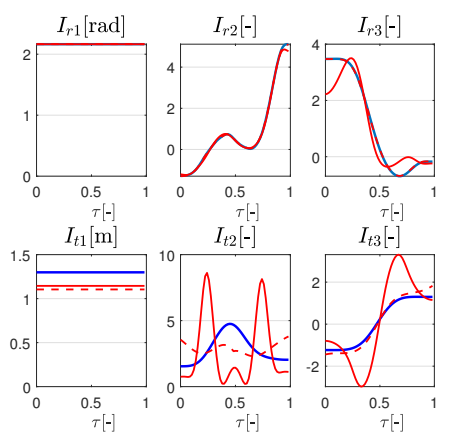

(c) eFSI shape descriptor

Figure 11: Adaptation (red) of the demonstrated motion trajectory (blue) while taking the workspace limits of a robot manipulator into account. In (a) the inverse kinematics step has the effect of projecting the trajectory on the workspace boundary, while in (b) this is avoided by directly including the robot kinematic model in the optimization, (c) yielding a reduced shape distortion.

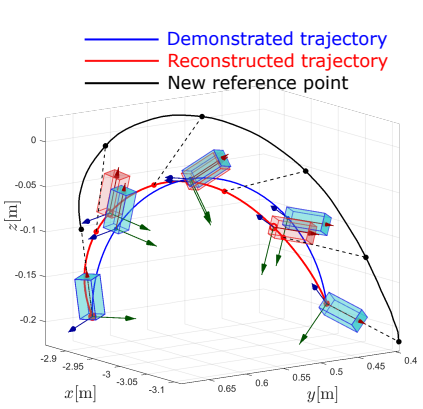

(a) Trajectory changes with eFSI

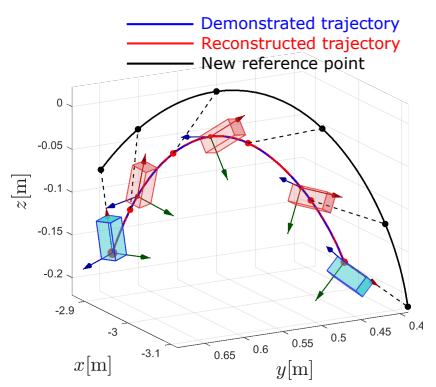

(c) Trajectory changes with SAI

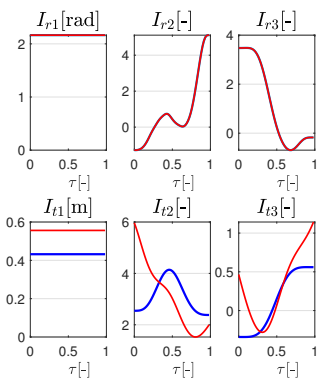

(b) eFSI shape descriptor
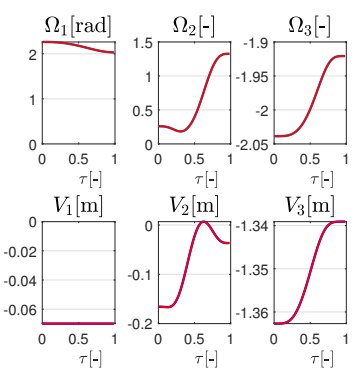

(d) SAl shape descriptor

Figure 12: The demonstrated trajectory (blue) is reconstructed (red) from its shape descriptor for the same initial and target pose, but the object's reference point for translation has been changed to another point on the object (black). The reconstructed trajectory and invariants do not change for the SAI shape descriptor thanks to its invariant properties for the choice of reference point, compared to the eFSI shape descriptor.

order to obtain smooth trajectories. The jerk-accuracy optimal control problem was chosen to be implemented and solved similarly to our optimization method by discretizing the dynamics equations assuming a constant jerk model, to allow for a better comparison. The jerk corresponds to the control inputs in the optimal control problem. The weight parameter $\lambda$ in [28] is chosen as 2.5 .

Table 3

Avera ge time to calculate the whole new trajectory for the three trajectory adaptation methods in the considered example.

\begin{tabular}{l|c} 
method & time $[\mathrm{s}]$ \\
\hline Jerk-accuracy optimization & 0.0276 \\
Dynamic movement primitives & 0.0229 \\
Shape descriptor optimization & 0.257
\end{tabular}

Figure 13a shows the generated trajectories (in red) for changes in target position. For the jerk-accuracy model, the new trajectories initially remain close to the demonstrated one (in blue) in order to keep the accuracy cost low. Later on, the trajectory transitions to an approximately straight line motion since the accuracy error between the generated trajectory and the demonstration becomes more and more important. When the target position is close to the demonstration, the result is fine, but farther away from the demonstration the shape becomes distorted. The corresponding shape descriptors of the generated trajectories are shown in Figure $13 \mathrm{~b}$ (using extended Frenet-Serret invariants) and provide a quantitative measure of this shape distortion.

\subsubsection{Dynamic movement primitives}

The Dynamic Movement Primitives (DMP) [21] represent trajectories as a dynamical system consisting of a global attractor to the target position and a non-linear force term encoding the trajectory shape. Again only the translation is considered for the comparison here. For including the rotation, the approach of [31] can be followed using a quaternion representation. Figure 13c shows the generated trajectories (red) to four new target positions. The parameters of the DMPs, as defined in [21], were chosen as $\alpha_{z}=2, \beta_{z}=\alpha_{z} / 4$, $\alpha_{x}=\alpha_{z} / 3$, and $N=10$. In the neighborhood of the demonstrated trajectory (blue), the generated trajectory looks fine but farther away from the demonstration the shape becomes more and more distorted. This is also reflected in the shape descriptors of the generated trajectories in Figure 13d when compared to the shape descriptor of the demonstration. 


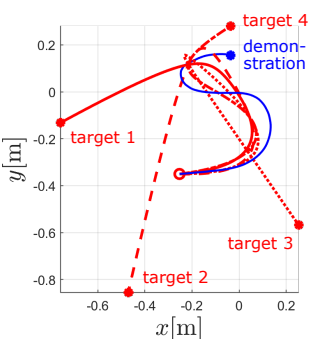

(a) Jerk-accuracy optimization

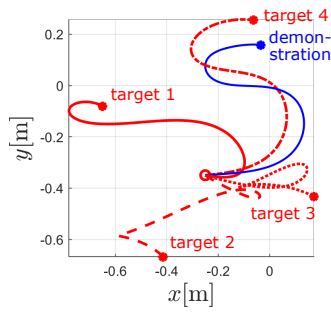

(c) Dynamic movement primitives

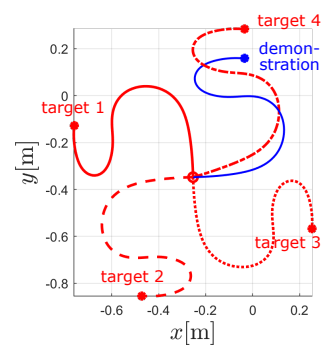

(e) Shape descriptor optimization

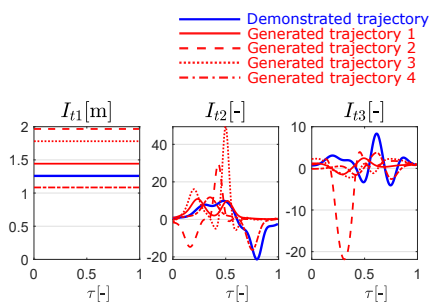

(b) eFSI of trajectories using jerk-accuracy optimization

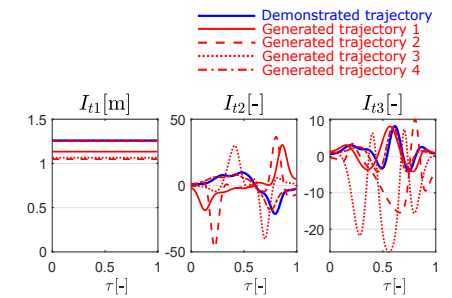

(d) eFSI of trajectories using dynamic movement primitives

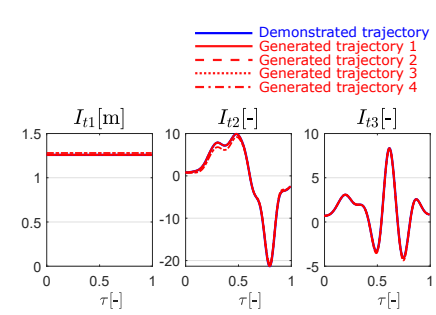

(f) eFSI of trajectories using shape descriptor optimization
Figure 13: Comparison between jerk-accuracy optimization, dynamic movement primitives, and shape descriptor optimization for changes in target position (red) with respect to the demonstration (blue).

\subsubsection{Coordinate-free shape descriptor optimization}

Generating the trajectories with the proposed constraintbased optimization method, where the extended Frenet-Serret invariants are the underlying shape descriptor, gives the results in Figure 13e. The generated trajectories (red) appear rotated in the plane. In this way, the shape remains more similar to the original demonstration (blue). This transformation is never explicitly stated, it follows from the specified constraints on the start and end position. The shape-similarity is also apparent by looking at the shape descriptors of the generated trajectories in Figure 13f, which remain closer to the shape descriptor of the demonstration than the other methods. This is expected since that is what we optimize for.

The purpose of these experiments was to show the specific invariant properties of the shape descriptor optimization method compared to the others. However, the other methods feature different qualities like faster calculation times. Table 3 shows there is an order of magnitude difference between the average calculation times of shape descriptor optimization with respect to jerk-accuracy optimization and dy- namic movement primitives. More on this in the discussion, in Section 6.2.

\section{Discussion}

The objective of this paper is to generalize demonstrated rigid-body trajectories to novel situations starting from few or even a single demonstration. By calculating a coordinatefree shape descriptor of the demonstration, the resulting motion models become more general, since they become independent of the choice of measurement frame, object references, and the timing along the trajectory. New trajectories are generated using constraint-based optimization where new task requirements are added as constraints and deviation with respect to the shape descriptor of the demonstration is minimized to preserve similarity.

Results indicate excellent generalization properties thanks to the shape descriptor approach, since changes in location and direction in trajectory space have no influence on the generated trajectory if the trajectory shape remains the same. When new task constraints require changes in the shape, these changes are tried to be kept as minimal as possible. Two existing shape descriptors are compared in the experiments: the extended Frenet-Serret invariant descriptor (eFSI) and the screw axis invariant descriptor (SAI). The main practical difference between the two is that the SAI are independent for the choice of reference point on the object for the translation, as shown in Figure 12. This means that the same motion trajectory will be calculated if the reference point changes, which may occur when the robot grasps a different part of the object or when the object is replaced with another. However, if the reference point of the demonstration is known, it is easy for the eFSI to recompute the trajectory for the new reference point. In case the robot always needs to handle the same object and grasp it in the same way as in the demonstrations, it is easier to use the eFSI shape descriptor than the SAI descriptor.

\subsection{Metrics for generalization and optimality solution}

The ultimate goal in trajectory generalization is to generalize similarly as humans do. In general, there exists no quantitative metric for evaluating how well a method generalizes demonstrated trajectories to new situations. The underlying metric this paper assumes is that the distortion in trajectory shape with respect to the original demonstration should be as small as possible, quantified by the difference between invariants in the shape descriptor. This does not guarantee that a human-like motion will be produced. However, keeping the motion similar in shape to the original demonstration, regardless of the chosen coordinate frame and timing information, is obviously a desirable feature.

To preserve the shape, other approaches [14, 20, 12] apply a global coordinate transformation (rotation, translation, scaling) in a pre-processing step in order to change to new initial and final positions, which also preserves the shape. This transformation is not always uniquely defined though or may be undefined. An advantage of our approach is that 
these types of transformations do not need to be explicitly calculated, but follow implicitly from the given task constraints. Another practical advantage for learning trajectories is that no time-consuming calibration is required prior to measuring the demonstrations, since the resulting shape descriptor will be the same regardless of the chosen reference frames.

Different types of norms are available to measure the distance between the shape descriptors in the objective function (11a). Here, the squared $l_{2}$ norm was chosen. Besides the more favorable mathematical properties in optimization, the squared $l_{2}$ norm has a physical interpretation for some invariant functions in the shape descriptors. For example, since the geometric Frenet-Serret invariants for translation correspond to length, curvature, and torsion, the objective function can be interpreted as the total deformation energy that is needed to lengthen, bend, and twist the demonstrated trajectory to the generated trajectory. The weights in the objective function are then interpreted as the corresponding stiffness parameters. During generalization, the objective is to minimize the necessary deformation energy. For other invariants in the shape descriptor, this physical interpretation is unfortunately not as intuitive.

The solution of the optimization problem, and therefore the generated trajectory, depends on the choice of weight values $W$ in the objective function. In choosing these weights, there remains some freedom to explore the solution space and choose the best generalized trajectory depending on the specific needs of the intended application. While we do not give concrete guidelines for setting the weight values, the formulation of the proposed optimization method is a major contribution on its own, since, for a given choice of weights, it is guaranteed to provide a solution that is invariant for the choice of coordinate frame in which the task constraints are expressed and the choice of coordinate frame in which the demonstrations were measured.

\subsection{Limitations approach and comparison with state-of-the-art}

The main limitation of the approach is that a nonlinear optimization problem needs to be solved, requiring multiple iterations to converge to a solution. When the number of constraints increases, the number of iterations also increases. Even so, the generated trajectories are calculated fast enough to be considered for on-line use, as indicated in Table 2. Reducing the number of optimization variables, for example by reducing the number of samples (which does not detract much from the quality of the trajectory), and optimizing the code for on-line use (e.g. with model predictive control) will yield even shorter calculation times.

If generalization is only required close to the set of demonstrations another trajectory adaptation method would probably be more suitable, such as the dynamic movement primitives [21] or jerk-accuracy optimal control [28], since these methods calculate new trajectories faster (see Table 3). The proposed shape descriptor optimization approach on the other hand is preferred when few demonstrations are available and the new task constraints differ largely from the original set of demonstrations, requiring a drastic adaptation. Shape descriptor optimization then guarantees that the generalized trajectory is still similar to the demonstration.

Combining the shape descriptor method with other trajectory adaptation methods may yield the advantages of both. At the start of motion, shape descriptor optimization could generate a global trajectory taking different task constraints into account, while another method could provide real-time adaptations to small changes during execution. This promising combination of approaches will be the subject of future work.

\subsection{Application to more realistic scenarios}

Formulating the trajectory generalization problem using constrained optimization has the advantage that other task constraints are easy to include. Section 5.2 already worked out some simple scenarios, e.g. robot workspace limits and obstacle avoidance. More complex applications become feasible by combining multiple constraints from various sensors. Existing constraint-based task specification frameworks and corresponding controllers such as [2] may be used for this purpose, where minimizing shape descriptor deviation would then just be another constraint.

\section{Conclusion}

Coordinate-free trajectory shape descriptors can represent demonstrated trajectories independently of the chosen references during recording, such as the coordinate frames and timing. This paper builds on top of the generative properties of different shape descriptors for rigid-body trajectories and proposes a constraint-based optimization method for generalizing demonstrated trajectories to new situations, starting from the shape descriptor of the demonstration. Experiments show how even a single demonstrated trajectory is successfully generalized to new situations, such as new initial and target positions, including obstacle avoidance and robot platform constraints, while preserving similarity with the original demonstration. These results encourage the use of shape descriptors for generalization in learning by demonstration applications, since fewer demonstrations are required to construct generally valid motion models.

\section{Acknowledgments}

All authors gratefully acknowledge the financial support by the Flemish FWO project G0B6613, the Flanders Make project Yves and the ERC Advanced Grant: ROBOTGENSKILL.

The KU Leuven Robotics Research Group is a Core Lab of Flanders Make.

\section{References}

[1] Abderrahim, M., Khamis, A., Garrido, S., Moreno, L., 2006. Accuracy and calibration issues of industrial manipulators, in: Industrial Robotics: Programming, Simulation and Applications. IntechOpen. chapter 7, pp. 131-146. 
[2] Aertbeliën, E., De Schutter, J., 2014. etas1/etc: A constraint-based task specification language and robot controller using expression graphs, in: 2014 IEEE/RSJ International Conference on Intelligent Robots and Systems, pp. 1540-1546. doi:10.1109/IROS. 2014.6942760.

[3] Aleotti, J., Caselli, S., 2006. Robust trajectory learning and approximation for robot programming by demonstration. Robotics and $\mathrm{Au}-$ tonomous Systems 54, 409-413.

[4] Andersson, J., 2013. A General-Purpose Software Framework for Dynamic Optimization. PhD thesis. Arenberg Doctoral School, KU Leuven. Department of Electrical Engineering (ESAT/SCD) and Optimization in Engineering Center, Kasteelpark Arenberg 10, 3001Heverlee, Belgium.

[5] Argall, B.D., Chernova, S., Veloso, M., Browning, B., 2009. A survey of robot learning from demonstration. Robotics and autonomous systems 57, 469-483.

[6] Asfour, T., Azad, P., Gyarfas, F., Dillmann, R., 2008. Imitation learning of dual-arm manipulation tasks in humanoid robots. International Journal of Humanoid Robotics 5, 183-202.

[7] Bennequin, D., Fuchs, R., Berthoz, A., Flash, T., 2009. Movement timing and invariance arise from several geometries. PLoS Comput Biol 5, 1-27. doi:10.1371/journal. pcbi. 1000426.

[8] Biess, A., Liebermann, D.G., Flash, T., 2007. A computational model for redundant human three-dimensional pointing movements: integration of independent spatial and temporal motor plans simplifies movement dynamics. Journal of Neuroscience 27, 13045-13064.

[9] Billard, A., Calinon, S., Dillmann, R., Schaal, S., 2008. Robot programming by demonstration, in: Springer handbook of robotics. Springer, pp. 1371-1394.

[10] Boyd, S., Vandenberghe, L., 2004. Convex Optimization. Cambridge University Press, New York, NY, USA.

[11] Calinon, S., 2016. A tutorial on task-parameterized movement learning and retrieval. Intelligent Service Robotics 9, 1-29. doi:10.1007/ s11370-015-0187-9.

[12] Calinon, S., Alizadeh, T., Caldwell, D.G., 2013. On improving the extrapolation capability of task-parameterized movement models, in: Proc. IEEE/RSJ Intl Conf. on Intelligent Robots and Systems (IROS), Tokyo, Japan. pp. 610-616.

[13] Calinon, S., Guenter, F., Billard, A., 2007. On learning, representing, and generalizing a task in a humanoid robot. Systems, Man, and Cybernetics, Part B: Cybernetics, IEEE Transactions on 37, 286-298.

[14] Cohen, Y., Berman, S., 2013. Tight dynamic movement primitives for complex trajectory generation, in: 2013 IEEE International Conference on Systems, Man, and Cybernetics, pp. 2402-2407. doi:10. 1109/SMC. 2013.410.

[15] De Laet, T., Bellens, S., Smits, R., Aertbelien, E., Bruyninckx, H., De Schutter, J., 2013. Geometric relations between rigid bodies (part 1): Semantics for standardization. IEEE Robotics Automation Magazine 20, 84-93. doi:10.1109/MRA. 2012.2205652.

[16] De Schutter, J., 2010. Invariant description of rigid body motion trajectories. Journal of Mechanisms and Robotics 2, 011004/1-9.

[17] Diehl, M., Bock, H.G., Diedam, H., Wieber, P.B., 2006. Fast direct multiple shooting algorithms for optimal robot control, in: Fast motions in biomechanics and robotics. Springer, pp. 65-93.

[18] Friedrich, H., Mnch, S., Dillmann, R., Bocionek, S., Sassin, M., 1996. Robot programming by demonstration (rpd): Supporting the induction by human interaction. Machine Learning 23, 163-189.

[19] Gilbert, E.G., Johnson, D.W., Keerthi, S.S., 1988. A fast procedure for computing the distance between complex objects in threedimensional space. IEEE Journal on Robotics and Automation 4, 193-203. doi:10.1109/56. 2083.

[20] Gräve, K., Behnke, S., 2012. Incremental action recognition and generalizing motion generation based on goal-directed features, in: Intelligent Robots and Systems (IROS), 2012 IEEE/RSJ International Conference on, IEEE. pp. 751-757.

[21] Ijspeert, A.J., Nakanishi, J., Hoffmann, H., Pastor, P., Schaal, S., 2013. Dynamical movement primitives: learning attractor models for motor behaviors. Neural computation 25, 328-373.

[22] Khansari-Zadeh, S.M., Billard, A., 2011. Learning stable nonlinear dynamical systems with gaussian mixture models. Robotics, IEEE Transactions on 27, 943-957.

[23] Kim, S., Haschke, R., Ritter, H., 2017. Gaussian mixture model for 3-dof orientations. Robotics and Autonomous Systems 87, 28 - 37. doi:https://doi.org/10.1016/j. robot.2016.10.002.

[24] Kramberger, A., Gams, A., Nemec, B., Chrysostomou, D., Madsen, O., Ude, A., 2017. Generalization of orientation trajectories and force-torque profiles for robotic assembly. Robotics and Autonomous Systems 98, 333 - 346. doi:https://doi .org/10.1016/j. robot. 2017. 09.019.

[25] Kühnel, W., 2006. Differential geometry: curves-surfaces-manifolds. volume 16. American Mathematical Soc.

[26] Lee, D., Soloperto, R., Saveriano, M., 2018. Bidirectional invariant representation of rigid body motions and its application to gesture recognition and reproduction. Autonomous Robots 42, 125-145. doi:10.1007/s10514-017-9645-x.

[27] Lemme, A., Meirovitch, Y., Khansari-Zadeh, S.M., Flash, T., Billard, A., Steil, J.J., 2015. Open-source benchmarking for learned reaching motion generation in robotics. Paladyn, J. Behav. Robot. 6, 30-41.

[28] Meirovitch, Y., Bennequin, D., Flash, T., 2016. Geometrical invariance and smoothness maximization for task-space movement generation. IEEE Transactions on Robotics 32, 837-853.

[29] Murray, R.M., Sastry, S.S., Zexiang, L., 1994. A Mathematical Introduction to Robotic Manipulation. 1st ed., CRC Press, Inc., Boca Raton, FL, USA.

[30] Paraschos, A., Daniel, C., Peters, J., Neumann, G., 2013. Probabilistic movement primitives, in: Advances in Neural Information Processing Systems, pp. 2616-2624.

[31] Pastor, P., Righetti, L., Kalakrishnan, M., Schaal, S., 2011. Online movement adaptation based on previous sensor experiences, in: IEEE/RSJ International Conference on Intelligent Robots and Systems, IEEE. pp. 365-371.

[32] Pekarovskiy, A., Nierhoff, T., Hirche, S., Buss, M., 2018. Dynamically consistent online adaptation of fast motions for robotic manipulators. IEEE Transactions on Robotics 34, 166-182. doi:10.1109/TRO. 2017.2765666.

[33] Pham, Q.C., Nakamura, Y., 2015. A new trajectory deformation algorithm based on affine transformations. IEEE Transactions on Robotics 31, 1054-1063. doi:10.1109/TRO.2015.2450413.

[34] Ramirez-Amaro, K., Beetz, M., Cheng, G., 2017. Transferring skills to humanoid robots by extracting semantic representations from observations of human activities. Artificial Intelligence 247, 95-118.

[35] Santos, R.F., Pereira, G.A., Aguirre, L., 2018. Learning robot reaching motions by demonstration using nonlinear autoregressive models. Robotics and Autonomous Systems 107, 182 - 195. doi:https: //doi.org/10.1016/j.robot.2018.06.006.

[36] Saunders, J., Nehaniv, C.L., Dautenhahn, K., 2006. Teaching robots by moulding behavior and scaffolding the environment, in: Proceedings of the 1st ACM SIGCHI/SIGART conference on Human-robot interaction, ACM. pp. 118-125.

[37] Schulman, J., Duan, Y., Ho, J., Lee, A., Awwal, I., Bradlow, H., Pan, J., Patil, S., Goldberg, K., Abbeel, P., 2014. Motion planning with sequential convex optimization and convex collision checking. Int. J. Rob. Res. 33, 1251-1270. doi:10.1177/0278364914528132.

[38] Soloperto, R., Saveriano, M., Lee, D., 2015. A bidirectional invariant representation of motion for gesture recognition and reproduction, in: 2015 IEEE International Conference on Robotics and Automation (ICRA), pp. 6146-6152. doi:10.1109/ICRA. 2015.7140062.

[39] Ude, A., Atkeson, C.G., Riley, M., 2000. Planning of joint trajectories for humanoid robots using b-spline wavelets, in: Proceedings 2000 ICRA. Millennium Conference. IEEE International Conference on Robotics and Automation. Symposia Proceedings (Cat. No.00CH37065), pp. 2223-2228 vol.3. doi:10.1109/ROBOT. 2000. 846358.

[40] Vakanski, A., Mantegh, I., Irish, A., Janabi-Sharifi, F., 2012. Trajectory learning for robot programming by demonstration using hidden markov model and dynamic time warping. Systems, Man, and Cybernetics, Part B: Cybernetics, IEEE Transactions on 42, 1039-1052. 
[41] Vochten, M., De Laet, T., De Schutter, J., 2015. Comparison of rigid body motion trajectory descriptors for motion representation and recognition, in: 2015 IEEE International Conference on Robotics and Automation (ICRA), pp. 3010-3017. doi:10.1109/ICRA.2015.7139612.

[42] Vochten, M., De Laet, T., De Schutter, J., 2016. Generalizing demonstrated motions and adaptive motion generation using an invariant rigid body trajectory representation, in: 2016 IEEE International Conference on Robotics and Automation (ICRA), pp. 234-241. doi:10.1109/ICRA. 2016.7487139.

[43] Vochten, M., Laet, T.D., Schutter, J.D., 2018. Robust optimizationbased calculation of invariant trajectory representations for point and rigid-body motion, in: 2018 IEEE/RSJ International Conference on Intelligent Robots and Systems, IROS 2018, Madrid, Spain, October 1-5, 2018, IEEE. pp. 5598-5605. doi:10.1109/IROS.2018.8593540.

[44] Wächter, A., Biegler, L.T., 2006. On the implementation of an interior-point filter line-search algorithm for large-scale nonlinear programming. Mathematical Programming 106, 25-57. doi:10.1007/ s10107-004-0559-y.

[45] Wu, S., Li, Y.F., 2009. Flexible signature descriptions for adaptive motion trajectory representation, perception and recognition. Pattern Recognition 42, 194-214. 